\title{
Traslocación de la costra liquénica en ambientes yesíferos \\ semiáridos mediante uso de adhesivos
}

Joaquín Ayerbe López

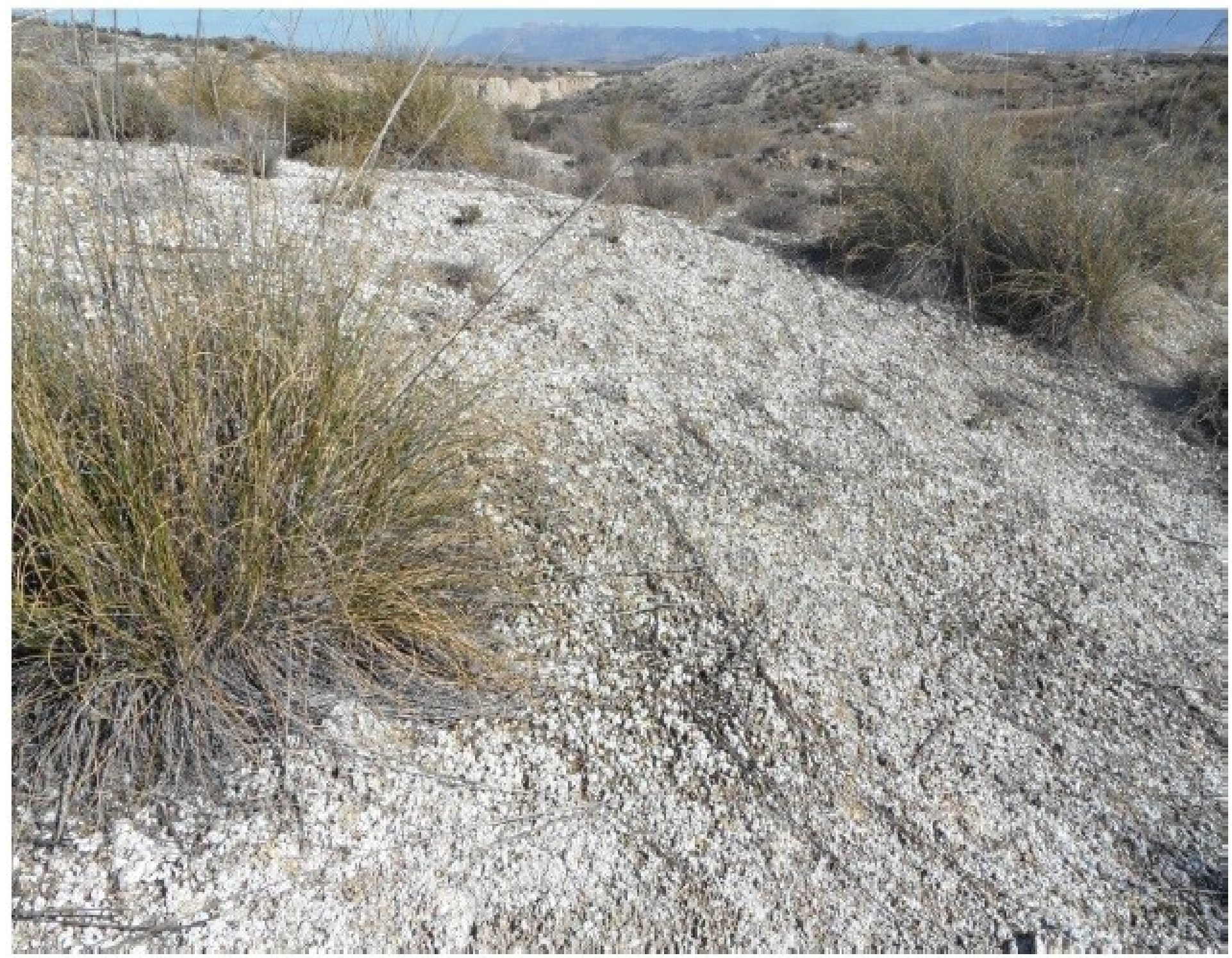

Trabajo Fin de Máster

Máster Oficial en Conservación, Gestión y Restauración de la Biodiversidad 


\title{
TRASLOCACIÓN DE LA COSTRA LIQUÉNICA EN AMBIENTES YESÍFEROS SEMIÁRIDOS MEDIANTE USO DE ADHESIVOS
}

\author{
MÁSTER EN CONSERVACIÓN, RESTAURACIÓN Y GESTIÓN DE LA \\ BIODIVERSIDAD
}

TRABAJO FIN DE MÁSTER

Joaquín Ayerbe López

TUTORES: Juan Lorite Moreno

Manuel Casares Porcel

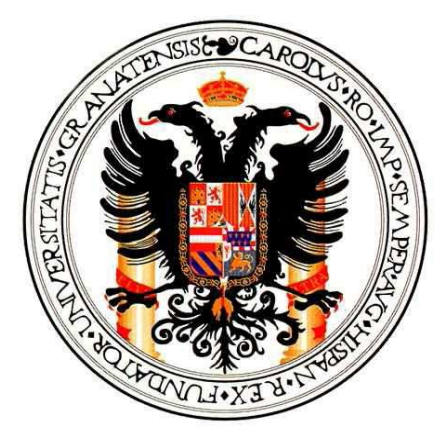

ugr

\section{Universidad de Granada}




\section{ÍNDICE}

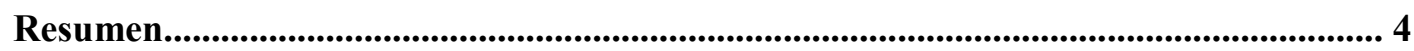

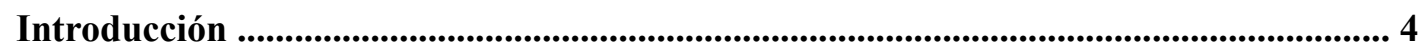

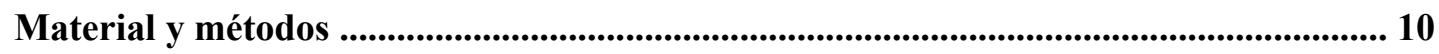

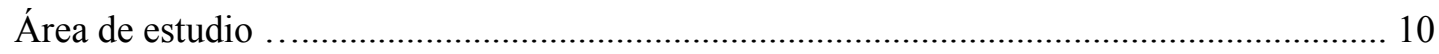

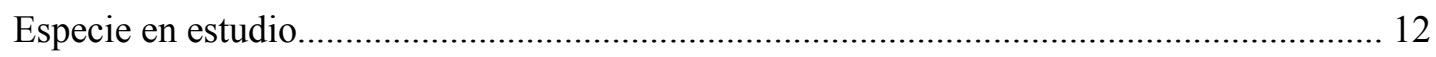

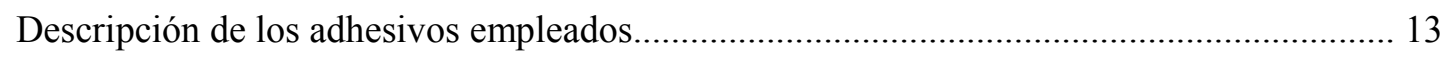

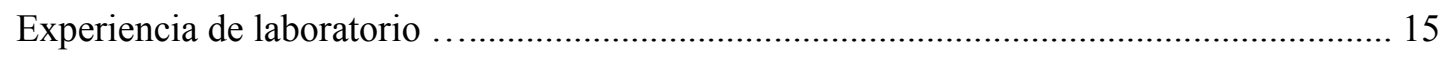

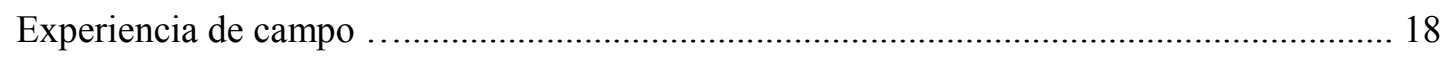

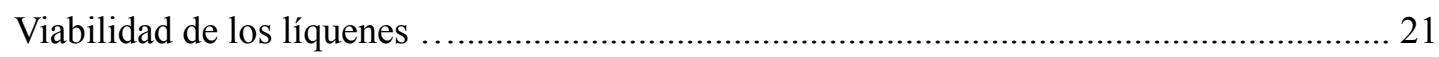

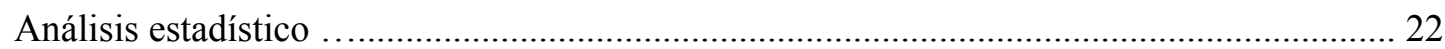

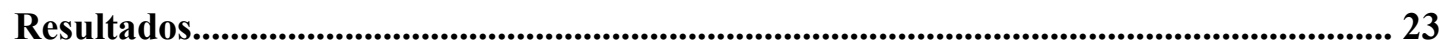

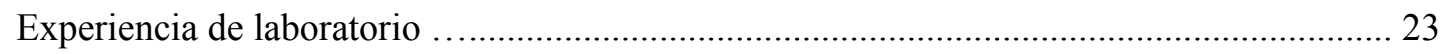

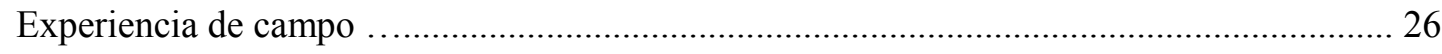

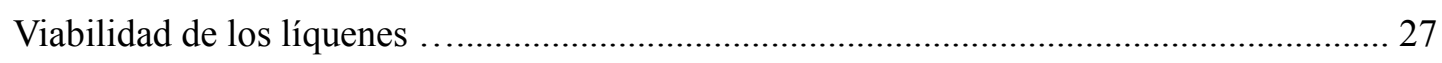

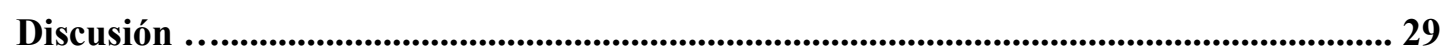

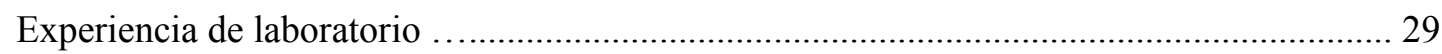

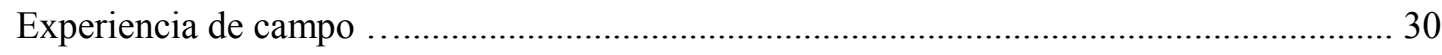

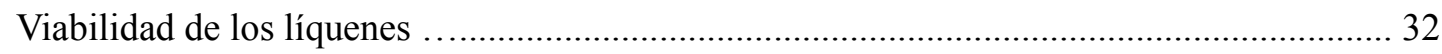

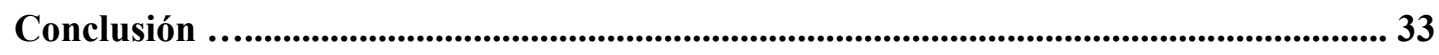

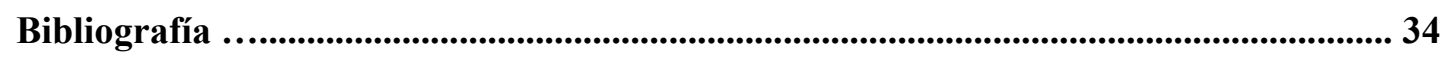

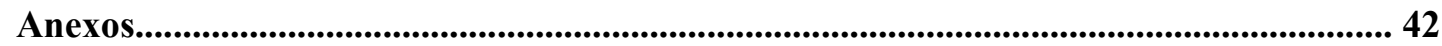

Agradecimientos................................................................................................................................... 44 


\section{RESUMEN}

La pérdida de la costra biológica debido a la explotación del suelo para la obtención de minerales (canteras), supone un problema ambiental ampliamente extendido; además, su restauración supone un reto considerable en aquellas zonas con una composición singular en cuanto al sustrato, como son las zonas yesíferas, donde los componentes de dichas costras y en concreto los líquenes adquieren, si cabe, mayor singularidad. Son pocos los trabajos que abordan la restauración de los líquenes que forman parte de las costras biológicas. El área en la que se ha desarrollado el estudio, se encuentra en una localidad al sureste de la Península Ibérica marcada por su clima mediterráneo continental. Hemos evaluado la eficacia de adhesivos de diferente naturaleza para su uso tras una traslocación de los líquenes a una hipotética zona de restauración de la costra liquénica, analizando su efectividad en cuanto a la capacidad para mantener los talos traslocados fijos en el sustrato y valorando (mediante medidas de respiración) los posibles efectos que los adhesivos pueden producir sobre la funcionalidad biológica de los líquenes. El ensayo se llevó a cabo con líquenes de la especie Diploschistes diacapsis que es predominante en el área de estudio. Finalmente se observó que los adhesivos que mejores resultados producían eran el agua y el pegamento de hidrosiembra, debido a su capacidad de mantener los talos de líquen adheridos al sustrato durante un periodo de tiempo prolongado sin causar ninguna alteración significativa en sus funciones biológicas. Se pudo concluir además que los factores que causan un mayor desplazamiento de los talos liquénicos son en primer lugar el viento, seguido del agua en forma de precipitaciones.

\section{INTRODUCCIÓN}

A lo largo y ancho de las tierras emergidas del planeta los suelos ricos en yeso son muy representativos, encontrándose distribuidos de forma más o menos heterogénea. Gran parte de los afloramientos de yeso existentes proceden del Triásico, Jurásico o Cretácico y, predominantemente, de depósitos de yeso del Mioceno, los cuales suelen aparecer intercalados en margas o arcillas, ocupando una superficie de entre 100 y 150 millones de hectáreas aproximadamente (Driessen et Dudal, 1991), que se reparten por los cinco continentes (Imagen 1): al norte, este y sur de África, sur de Europa y zonas del centro de la antigua Unión Soviética, Oriente Medio, suroeste de Asia, sureste y centro de Australia, en México y el suroeste de Estados Unidos (Boyadgiev et Verheye, 1996). 


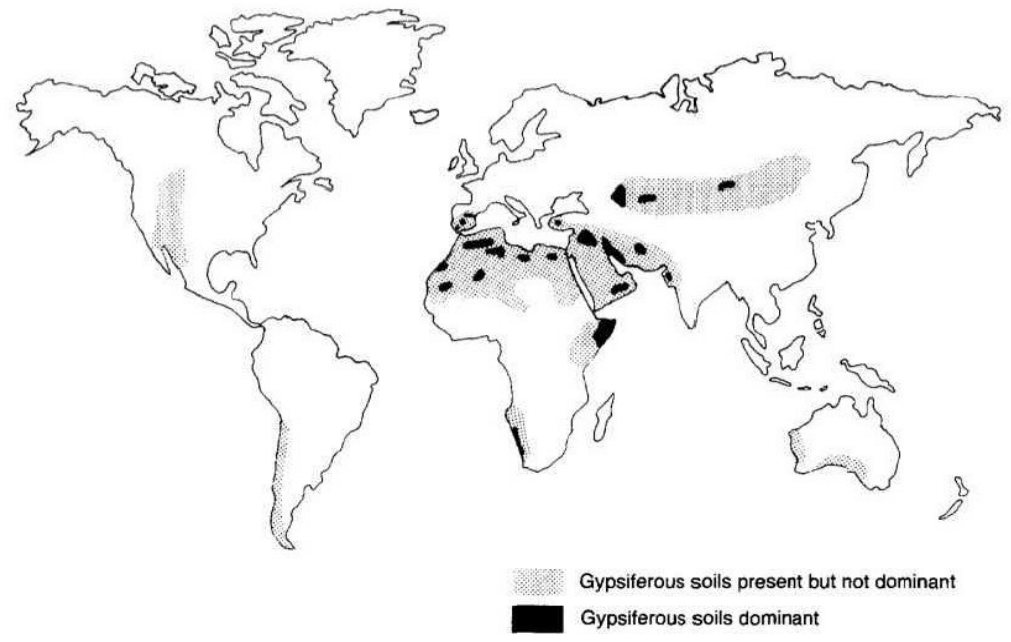

Imagen 1. Distribución de los suelos ricos en yesos por todo el mundo. (Fuente, Boyadgiev \& Verheye, 1996). Se observa como en la mitad sur de nuestro país son predominantes los suelos yesíferos.

Los suelos yesíferos en la Península Ibérica se encuentran distribuidos principalmente por la mitad oriental (Gil \& Ramos, 2011). Estos sustratos cubren una superficie aproximada de $35.487 \mathrm{~km}^{2}$, cerca del 12\% del territorio de España (Riba \& Macau, 1962), haciéndose patentes dos tipos de afloramientos de yesos: los que se desarrollan sobre materiales Triásicos y los yesos terciarios que corresponden a depósitos del Mesiniense (Gil \& Ramos, 2011). En este periodo (Mesiniense) se produjo una crisis de salinidad en el Mediterráneo produciéndose la aparición de cuencas hipersalinas aisladas, en las que se dieron procesos de evaporación, con la posterior deposición de las sales disueltas (Guerra et al, 1993) que son las que hoy encontramos en los suelos yesíferos de nuestro territorio. En la Península Ibérica encontramos cuatro grandes territorios de yesos: I) el valle del Ebro, II) sureste Andaluz (yesos almerienses), III) sureste peninsular (yesos murcianos y alicantinos) y IV) una gran extensión territorial que cubre gran parte del centro peninsular y áreas circundantes correspondientes a Castilla y León y centro-oeste de Andalucía (depresión del Guadiana Menor, valle del Guadalquivir y afloramientos suroccidentales de Granada, zona en la que se centra nuestro estudio), siendo éste último el más heterogéneo desde el punto de vista de su composición florística (Marchal et al, 2008).

Estos suelos ricos en yesos se encuentran en general por encima de los $600 \mathrm{msnm}$ y en el interior peninsular, lo que le confiere un clima continental con gran amplitud térmica tanto diaria como estacional. Los afloramientos superficiales tienden a estar asociados con zonas semiáridas, ya 
que en áreas con mayor pluviometría el yeso se disuelve (Casares-Porcel et al, 1996). En la provincia de Granada podemos encontrar dos grandes áreas yesíferas, la primera localizada en la zona más septentrional de la provincia (Hoya de Guadix y Baza) y la segunda localizada en la parte centro-occidental de la misma. Los principales afloramientos de yeso del centro-oeste de la provincia de Granada se sitúan al sur de las localidades de La Malahá, Escúzar y Ventas de Huelma, aunque también se han encontrado pequeños yacimientos más dispersos hacia al suroeste (Embalse de los Bermejales), y en la zona al norte de la capital (Alfacar), por último también encontramos afloramientos en las proximidades de Cacín (Lorite et al, 2011).

El desarrollo de una cobertura vegetal densa se hace difícil en los subdesiertos, y aún más cuando se desarrollan sobre sustratos ricos en yeso. Las precipitaciones escasas e irregularmente distribuidas a lo largo del año, el limitado desarrollo del suelo, la presencia de yeso y la elevada evapotranspiración (como consecuencia de la fuerte insolación), que hace ascender las sales de las capas más profundas del suelo depositándose en la superficie, son factores que muy pocas especies vegetales están en condiciones de soportar (Casares-Porcel \& Gutiérrez-Carretero, 2004). Esta es la razón por la que en este tipo de ambientes se desarrolla una escasa cobertura vegetal, ya que se trata de un suelo poco productivo y estresante (Rubio \& Escudero, 2000). Este hecho hace de estos ambientes lugares singulares donde se desarrollan numerosas especies raras y/o endémicas, muchas de ellas amenazadas y en algunos casos protegidas legalmente (Bañares et al, 2004). Todo esto ha ocasionado que este tipo de hábitat esté recogido en la Directiva Hábitat, como prioritario para la conservación (Anónimo, 1992).

Por otro lado es necesario considerar que los yesos son un importante recurso económico, ocasionando en determinadas zonas la aparición de actividades encaminadas a la explotación del mismo. De esta forma su extracción en canteras, que en ocasiones pueden ocupar amplias extensiones de terreno, supone un gran impacto a muchos niveles y supone un conflicto de intereses entre el aprovechamiento del recurso y la conservación del hábitat (Mota et al, 2004). Por todo lo expuesto anteriormente, en estos ambientes es muy necesario tomar medidas activas de restauración y en este sentido se han llevado a cabo algunos trabajos de investigación (e.g. Matesanz et Valladares, 2007; Castillejo et Castelló, 2010; Ballesteros et al, 2012; 2014). Todos estos trabajos se han centrado en plantas vasculares, concretamente en especies del matorral. 
Es necesario reseñar que en estos ambientes semiáridos, entre los espacios que dejan las plantas vasculares, se establecen comunidades formadas por organismos autótrofos (cianobacterias, líquenes y musgos) y heterótrofos (hongos y bacterias), formando las denominadas costras biológicas (Foto 1), que frecuentemente resultan ser el elemento con mayor cobertura en el ecosistema (Belnap \& Gillette, 1998). Entre las propiedades más importantes que poseen estas costras biológicas están la de evitar la erosión eólica (Belnap \& Gillette, 1997) o hidráulica (Bowker, 2007), además de suponer una trampa de nutrientes, dando estabilidad al suelo (CastilloMonroy \& Maestre, 2011), confiriéndole más productividad gracias a la fijación de nitrógeno atmosférico por parte de los organismos que forman la costra (Harper \& Belnap, 2001).

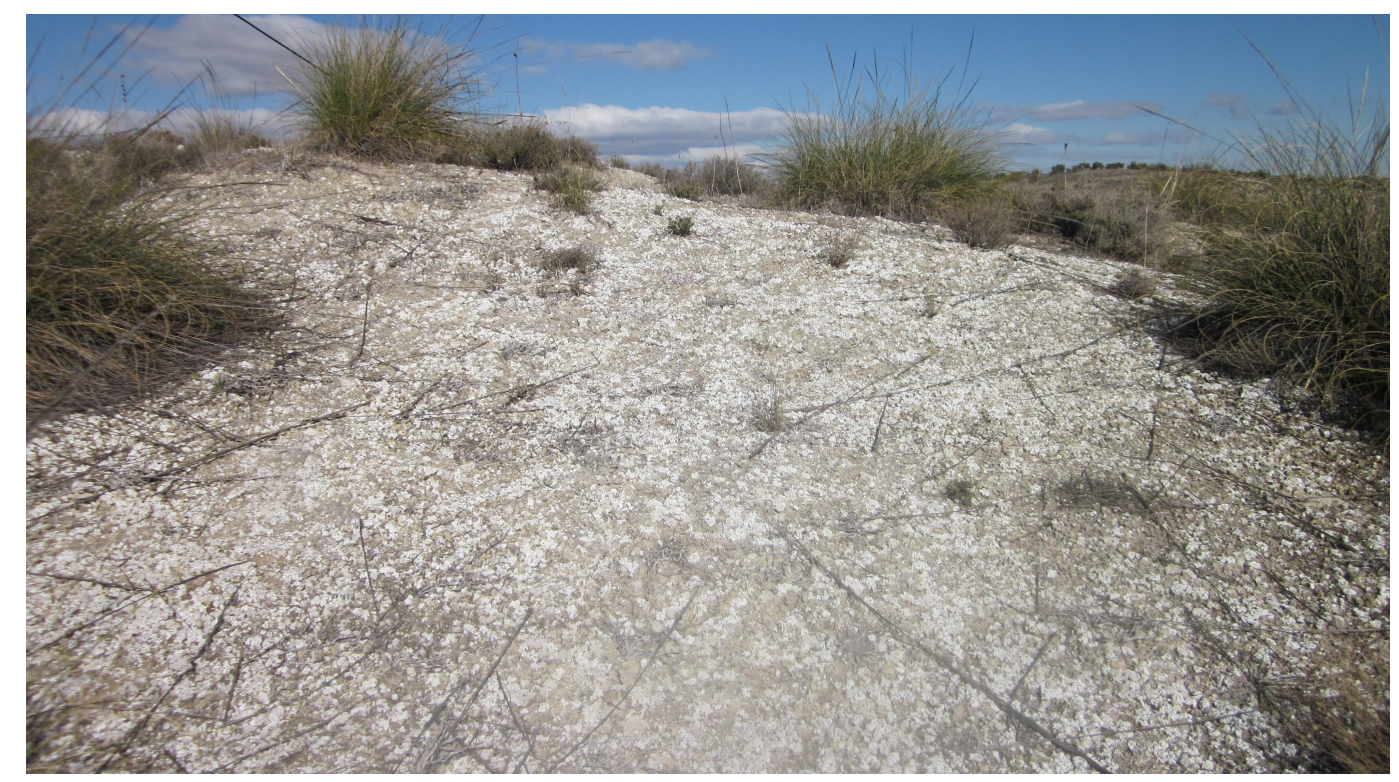

Foto 1. Detalle de un área cercana a la zona de estudio donde se observa claramente la costra biológica. En dicha costra (en el área de estudio) los talos de Diploschistes diacapsis alcanzan hasta un $80 \%$ de cobertura (Ibarz, 2012).

A parte de lo comentado anteriormente presentan un importante papel en el ciclo del carbono (Cable \& Huxman, 2004) e influyen notablemente en la disponibilidad de otros nutrientes (Harper $\&$ Belnap, 2001). Es por esto que para muchos autores estas costras resulten ser fundamentales para el correcto funcionamiento del ecosistema (Bowker et al, 2005). Todo esto justifica el hecho de que se tengan que realizar esfuerzos en su mantenimiento y protección (Bowker \& Belnap, 2008). 
Es importante en nuestro caso comentar que el componente más representativo de las costras biológicas son los líquenes (Bowker \& Belnap, 2008), ya que en ocasiones cubren de un 60\% a un $80 \%$ de la superficie de los sustratos donde hay afloramientos de yesos. Estos líquenes encuentran en los afloramientos de yeso unas condiciones ambientales ideales para sus requerimientos. Por un lado debido al mantenimiento de humedad y la cohesión del sustrato que aporta el yeso, y por otro porque se ven favorecidos por la ausencia de competencia por parte de otras plantas (GutiérrezCarretero \& Casares-Porcel, 2011). Los líquenes ejercen diferentes efectos positivos sobre la hidrología del terreno (Bowker, 2007) y sobre la germinación de las semillas (Deines et al, 2007).

Por otro lado cabe decir que los líquenes son capaces de mantener una fotosíntesis neta positiva en condiciones extremas que influye en la productividad del ecosistema (Green et al, 2008).

Algunos de los líquenes que habitan en estos ambientes, son además especies raras y/o amenazadas, siendo por lo tanto objeto preferente de cara a la conservación (Guerra et al, 1995). Por todo lo comentado anteriormente es lógico que haya un elevado número de investigaciones acerca de las costras biológicas (e.g. Castillo-Monroy \& Maestre, 2011). Por ello, un elevado número de autores han estudiado los líquenes característicos de los afloramientos yesíferos (Llimona, 1974; Crespo et al, 1980; Barreno \& Merino, 1981; Burgaz \& Seriña, 1986; Guerra et al., 1993; Casares-Porcel \& Gutiérrez-Carretero, 1993, 1996; Casares-Porcel, 1994; Martínez-Sánchez, 1994; Egea \& Alonso, 1996). Señalando muchos autores la importancia de preservar y restaurar las costras biológicas (Castillo-Monroy \& Maestre, 2011; Bowker \& Belnap, 2008; Guerra et al, 1995; Hilty, 2004).

Sin embargo, a pesar de la importancia que la costra liquénica presenta en el funcionamiento de los ecosistemas de los que forma parte, existen pocos estudios que aborden su restauración (Bowker, 2007; Heinken, 1999) y hasta donde conocemos, en ninguno de ellos se ha ensayado una metodología para restaurarla después de una alteración drástica, como por ejemplo nuestro caso, una cantera de yesos. La ausencia de trabajos de restauración en la literatura se debe principalmente a la percepción de que la recuperación de las costras biológicas (líquenes, musgos, cianobacterias) no es un objetivo alcanzable a corto plazo (Bowker et al. 2007). Aunque la lenta regeneración de las costras biológicas ha evitado que se implementen medidas para promover su recuperación, 
existen evidencias de que la intervención puede reducir la escala temporal haciéndola más manejable en el contexto de un proyecto de restauración (Grettarsdottir et al. 2004, Li et al. 2004, Bowker et al. 2007).

Es importante destacar el lento crecimiento de los talos liquénicos (Nash, 1996; Benedict, 1967) lo cual hace de la traslocación un instrumento básico para la restauración de la costra liquénica, acelerando de esta forma el proceso de regeneración de la misma. Uno de los aspectos a tener en cuenta en la traslocación es la fijación de los talos al sustrato ya que el crecimiento de las hifas que fijan el talo es extremadamente lento (Nash, 1996), suponiendo esto la necesidad de aplicar algún método de fijación artificial.

Por las características comentadas, la colonización natural es demasiado lenta para proporcionar una cubierta vegetal eficaz que controle la erosión del suelo provocados por las lluvias torrenciales, frecuentes en estas áreas (Nicolau, 1996; Mota et al., 2003, 2004).

Por todo lo comentado anteriormente, y dentro de un programa más amplio de investigaciones, es necesario ensayar con distintos tipos de adhesivos para fijar de forma efectiva los talos al suelo, manteniendo su viabilidad (evitando una disminución en su vitalidad o incluso la muerte del talo). Encontrar un adhesivo idóneo supondría una solución al problema de traslocar y posteriormente fijar las costras liquénicas al sustrato.

Este trabajo pretende contribuir a diseñar y ensayar una metodología para restaurar la costra liquénica en ambientes semiáridos que, en la medida de lo posible, sea extrapolable a otro tipo de ambientes. Con este fin, el objetivo de este estudio es determinar la aplicabilidad de distintos adhesivos en la traslocación de talos liquénicos en un contexto de restauración, evaluando su efecto en: a) condiciones de precipitación y pendiente controladas; b) condiciones naturales en un escenario potencial de restauración; y c) comprobar su efecto sobre la vitalidad de los líquenes. 


\section{MATERIAL Y MÉTODOS}

\section{Área de estudio}

El área de estudio está localizada sobre afloramientos de yeso al suroeste de la provincia de Granada, concretamente en la localidad de Escúzar $\left(37^{\circ} 2^{\prime} \mathrm{N}, 3^{\circ} 45^{\prime} \mathrm{W}\right)$. Se encuentra a $950 \mathrm{msnm}$ (Imagen 2).

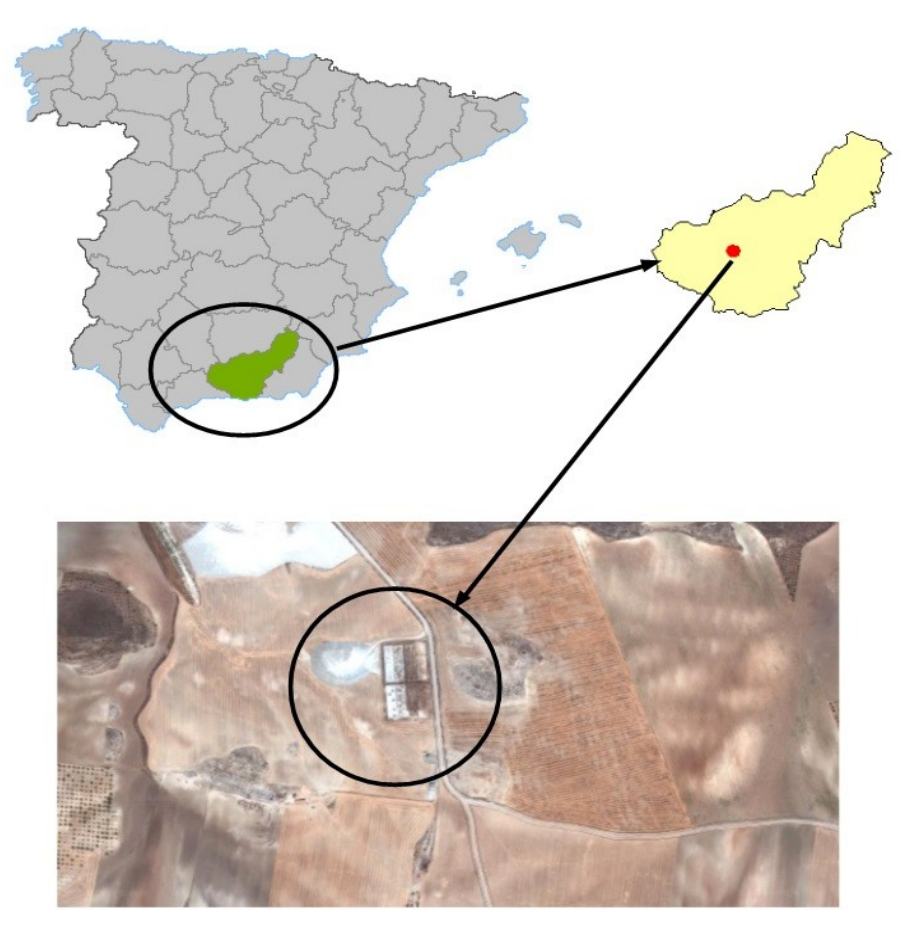

Imagen 2. Localización del área de estudio (Escúzar, Granada, $\mathrm{SE}$ de España). En la fotografía de abajo se observan en tonos más claros las distintas parcelas de experimentación.

El clima de la zona es Mediterráneo continental con veranos secos y calurosos e inviernos fríos y secos. Según los datos climáticos de la estación meteorológica más cercana de Padul (a 14 $\mathrm{km}$ de la zona de estudio, $37^{\circ} 01^{\prime} \mathrm{N}, 03^{\circ} 35^{\prime} \mathrm{W}$ ), la temperatura media anual registrada para la última década es de $15,5^{\circ} \mathrm{C}$, siendo la media del mes más frío (enero) de $7,5^{\circ} \mathrm{C}$ y la media del mes más cálido de $25{ }^{\circ} \mathrm{C}$ (agosto). Las épocas de lluvia están concentradas en otoño y primavera, siendo la precipitación media anual de $426 \mathrm{~mm}$, con un periodo de estrés hídrico de casi 6 meses al año. 
El territorio forma parte de la cuenca neógena de Granada y presenta depósitos de transición entre ambientes marinos y continentales, con niveles de halita y yeso. En concreto, en el área objeto de estudio afloran limos y yesos del Mioceno Superior (Vindoboniense) (Aldaya et al, 1980). Encima del yeso hay depósitos de margas lacustres, entre las que se intercalan niveles de arenas y conglomerados y en la base aparecen formaciones de origen marino. Contactan con calizas, margocalizas y limos lacustres con gasterópodos. Los suelos predominantes son regosoles, formados sobre rocas, en general, poco cementadas, que se caracterizan por presentar un perfil tipo $\mathrm{AC}$, su escasa evolución se debe a factores climáticos y/o a fenómenos erosivos. Existen en la zona diversos tipos de regosoles, todos ellos calcáreos o fuertemente calcáreos, como regosoles margálicos, regosoles litosólicos y calcáricos. Los suelos que nos interesan en nuestro estudio son los que aparecen sobre yesos, concretamente xerosoles gípsicos, en los que existe una acumulación de yeso cristalino en forma de macrocristales y eflorescencias cristalinas. Estos suelos se asocian fundamentalmente a climas áridos y semiáridos, y carecen de pedregosidad o bien es muy baja. Suelen presentan una textura franca, aumentando el limo con la profundidad debido a la influencia del material margoso. El contenido en materia orgánica es muy bajo en todo el perfil, así como el contenido en macronutrientes y la capacidad de cambio de cationes. El complejo de cambio de estos suelos está siempre saturado, siendo el calcio prácticamente el único catión presente. La cantidad de carbonatos es media y disminuye con la profundidad, de igual forma que el yeso (Aguilar et al, 1992).

La zona presenta parches aislados de vegetación natural (matorrales gipsícolas), que ocupan unos 4,7 $\mathrm{km}^{2}$ del territorio (Lorite et al, 2011). Las comunidades presentes en ellos son fundamentalmente: espartales (Helianthemo squamati-Stipetum tenacissimae) y romeralesmatorrales gipsícolas (Helianthemo squamati-Ononidetum crassifoliae) (Marchal et al, 2008). Acompañan a estas formaciones albardinales (Dactylido hispanicae-Lygeetum sparti), yesquerales (Phlomido lychnitidis-Brachypodietum ramosi), y pastizales vivaces de Stipa parviflora (Plantagini albicantis-Stipetum parviflorae). La ausencia o disminución del yeso da paso a espartales (Thymo gracilis-Stipetum tenacissimae) y tomillares (Thymo gracilis-Lavanduletum lanatae) calcícolas, incluso en alguna zona contactan con restos de encinar (Paeonio-Quercetum rotundifoliae) (Marchal et al., 2008). 
Entre los claros de las comunidades del espartal encontramos costras liquénicas, en algunos casos de extensión considerable (Foto 1), que dan al suelo un aspecto blanquecino que se confunde con los yesos. La asociación dominante de la zona es la Acarosporetum placodiiformi-reagentis Llimona 1974, perteneciente a la Alianza Psorion saviczii y que es la comunidad mejor representada en los terrenos yesíferos de la Península. Entre las especies más abundantes se pueden encontrar Diploschistes diacapsis (Ach.) Lumbsch, Acarospora placodiiformis H. Magn., Fulgensia desertorum (Tomin) Poelt y F. poeltii Llimona, siendo la dominante, Diploschistes diacapsis (Foto 2) que se caracteriza por presentar un grueso talo crustáceo de color blanco (Casares-Porcel \& Gutiérrez-Carretero, 1993).

\section{Especie en estudio}

Esta última especie se eligió para las experiencias por ser la que más cobertura y biomasa presentaba en el área de estudio; concretamente un $55 \%$ de cobertura y una biomasa en la costra liquénica superior al $80 \%$ (Ibarz, 2012).

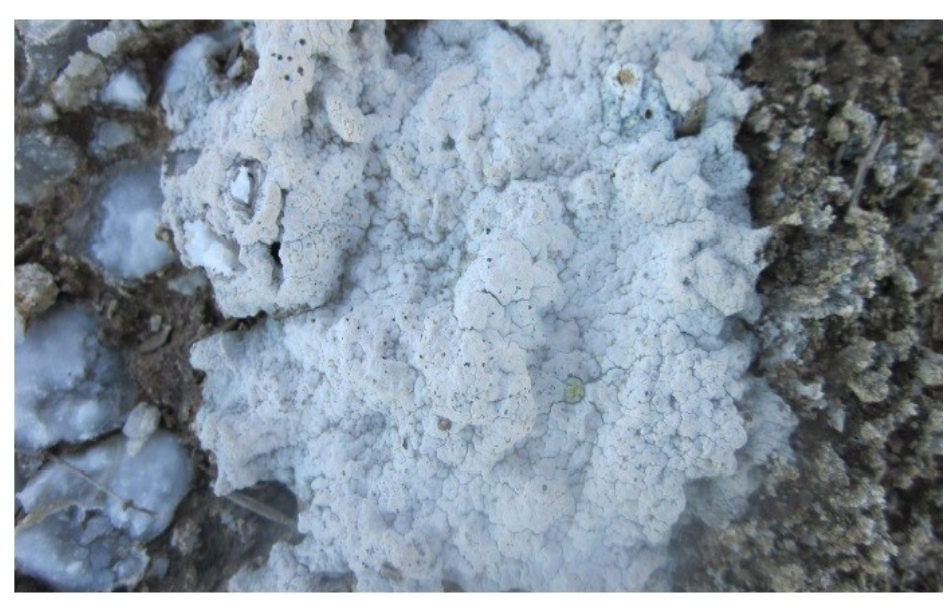

Foto 2. Talo balnco-grisáceo típico de Diposchistes diacapsis (Ach.) Lumbsch.

Por otro lado, la naturaleza del talo hace que esta especie sea bastante resistente a la manipulación y por lo tanto ideal para el experimento (donde se han tenido que troquelar los talos, manipular para aplicar el adhesivo, etc.). No obstante, los talos de esta especie tienen morfología y consistencia parecida a la de otras especies del área de estudio como por ejemplo: Acarospora nodulosa Dufour, Buellia zoharyi Galun, Cladonia foliacea (Huds.) Willd, Squamarina 
cartilaginea (With.) P. James o Fulgensia sp, entre otras. Por lo que los posibles resultados se podrían extrapolar a otras especies de la comunidad de líquenes del área de estudio.

Para los experimentos que se plantearon, se recogieron talos en la zona de estudio en otoño de 2013, concretamente en las inmediaciones de una cantera, en una zona que va a ser afectada por la extracción de mineral. Los talos se mantuvieron en sobres de papel, condiciones de laboratorio (ca. $22{ }^{\circ} \mathrm{C}, 15 \%$ de humedad), hasta la puesta en marcha de los distintos experimentos.

\section{Descripción de los adhesivos empleados}

A continuación se describen los adhesivos utilizados en los experimentos de laboratorio y campo, con el objetivo de determinar su efectividad, persistencia y efecto (positivo o negativo) sobre la viabilidad de los talos.

Se han utilizado 6 tratamientos, 5 adhesivos: 1) Pegamento de hidrosiembra (HS), 2) goma arábiga (AG), 3) resina sintética (SR), 4) cola blanca $(\mathrm{G})$ y 5) agua (W) (Foto 3), más un 6) control (C) sin adhesivo alguno. Con esta gama de adhesivos se pretendió utilizar materiales disponibles a nivel comercial, de naturaleza sintética y orgánica y utilizados frecuentemente o con una utilidad potencial en trabajos de restauración ecológica.

Foto 3. Detalle de los diferentes adhesivos que se valoraron para la experiencia, de los que finalmente fueron utilizados los que se detallan en el párrafo siguiente. Los adhesivos seleccionados fueron utilizados tanto en la experiencia de laboratorio, como en la de campo.

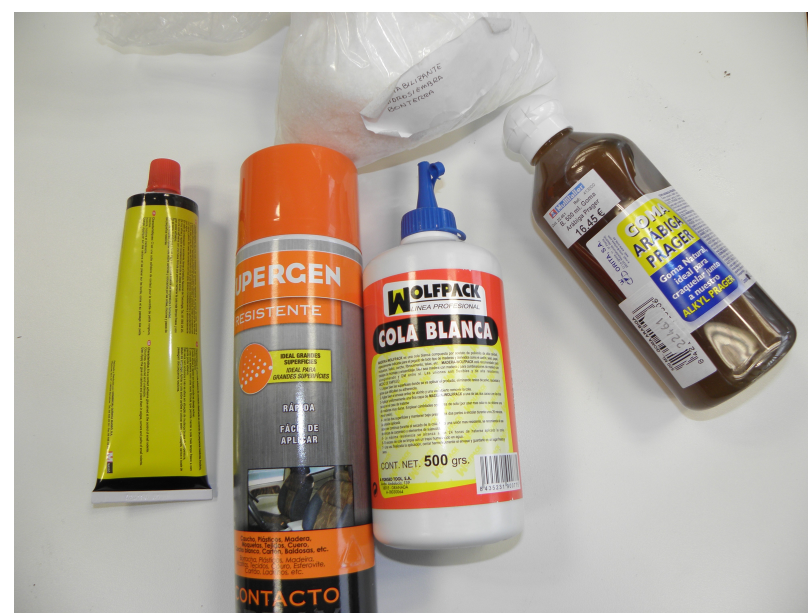


Descripción y justificación de la elección de los adhesivos:

- 1) Pegamento de hidrosiembra (BonTerra Ibérica), (HS): es una sustancia de uso común en trabajos de restauración para fijar las semillas al sustrato (Merlin, et al., 1999, GarcíaPalacios, et al., 2010), no es tóxico para organismos biológicos (según las indicaciones del fabricante) y proporciona humedad al objeto al que se adhiere. Se utilizó con una concentración de $4 \mathrm{~g} / \mathrm{L}$.

- 2) Goma arábiga (AG): es un adhesivo natural que se extrae de árboles de Acacia spp. Se trata de un polisacárido, no tóxico (según indicaciones del fabricante), ya que es utilizado como espesante en algunos alimentos, por lo que debería ser inocuo para los líquenes. Su uso ha sido sugerido como estabilizante para restauración de suelos (De Boodt, 1993)

- 3) Resina sintética (SR) fue seleccionada para el experimento por su resistencia a la desecación, por un lado y por su resistencia a la hidratación por otro (sigue siendo efectivo aunque se le aplique agua). Se desconocía si este tipo de adhesivo podía tener algún tipo de efecto dañino sobre la viabilidad de los líquenes.

- 4) Cola blanca $(G)$ : se eligió por su bajo coste y porque adhesivos parecidos han sido utilizados en experiencias similares, en las que se quería adherir talos (o tejidos vegetales) a diferentes sustratos (Gilbert, 1991; Smith, 2014). Se desconocía si el adhesivo tenía algún efecto negativo sobre los talos liquénicos.

- 5) Agua (W): Fue seleccionada como adhesivo por varias razones, aporta humedad al liquen en el momento de su aplicación, es barata, no es tóxica y por último al interaccionar con el sustrato (con alto contenido en yeso) actúa a modo de cementante, facilitando la inclusión del talo en el sustrato. Este tratamiento simula el proceso natural de adherencia de los fragmentos de talos, tras su dispersión por agentes abióticos (Heiken, 1999) como ocurriría en la naturaleza (Nash, 1996).. 


\section{Experiencia de laboratorio}

Con el experimento de laboratorio se ha tratado de analizar la capacidad de una serie de adhesivos de fijar los talos liquénicos en condiciones controladas de pendiente elevada y precipitación intensa.

Se usaron 30 bateas de plástico de $25 \times 35 \mathrm{~cm}$. ( 5 bateas réplicas x 6 tratamientos $=30$ ). A las bateas se les practicaron una serie de orificios ( 9 en la parte inferior y 10 en el lateral inferior) con el fin de facilitar el drenaje del agua durante la simulación de lluvia. Cada una de ellas fue rellenada con sustrato colectado en la zona de estudio (material de rechazo de la cantera de yeso) (Ver composición en Anexo 1). Este sustrato se pasó por un tamiz de $0,5 \mathrm{~cm}$ de luz de malla, obteniéndose un sustrato homogéneo con el que se rellenaron las bateas ( $2 \mathrm{~L} \mathrm{x}$ batea). Una vez depositado el sustrato en las bateas se regó para que presentara una cohesión y estabilidad adecuada, dejando secar antes de empezar la experiencia. En cada batea se colocaron diez talos de líquen procedentes de la zona de estudio con forma circular y con un diámetro de 1,5 cm., que fueron previamente troquelados con forma circular y con un diámetro de $1,5 \mathrm{~cm}$. para obtener unidades experimentales (Fotos 4 y 5). Los talos se colocaron en la zona central de las bateas, aplicando el tratamiento correspondiente, dispuestos en dos filas de 5, equidistantes entre ellos (Foto 6). El número total de talos para cada tratamiento fue de 50 (10 talos x 5 réplicas) por lo que el total para la experiencia ascendió a 300 talos (50 talos x 6 tratamientos adhesivos, incluido el control).

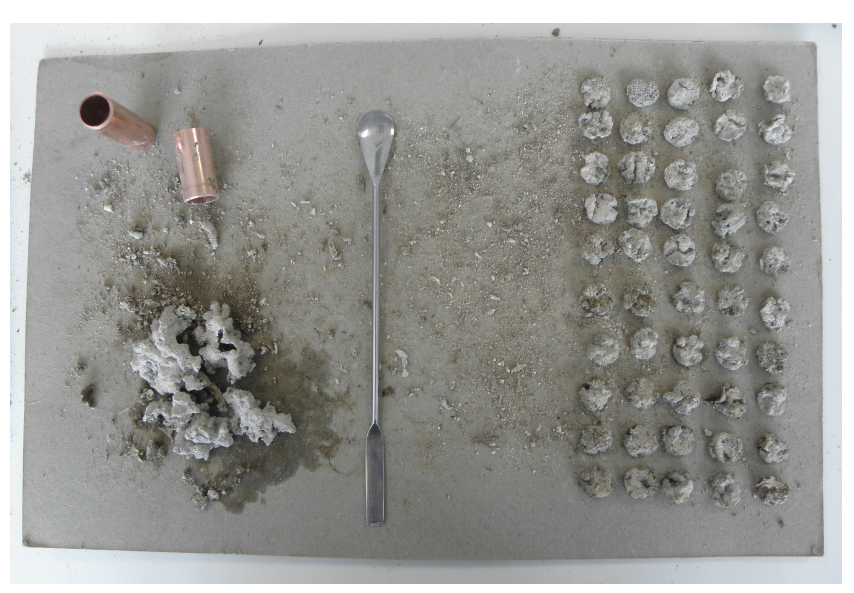

Foto 4. Procedimiento de obtención de los talos con forma redonda $(1,5 \mathrm{~cm}$ de diámetro) para el que se utilizaron tubos metálicos convenientemente afilados. Los líquenes eran humedecidos antes de cortarlos para facilitar el proceso (arriba a la izquierda).

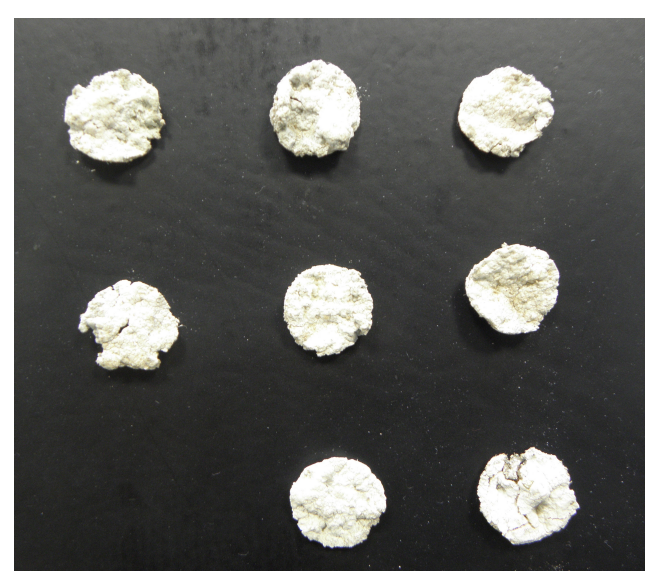

Foto 5. Conjunto de talos obtenidos mediante el procedimiento anteriormente descrito. (Arriba a la derecha) 
Foto 6. Batea preparada para la experiencia con el sustrato y los 10 talos de líquenes en dos filas de 5 colocados en la parte central.

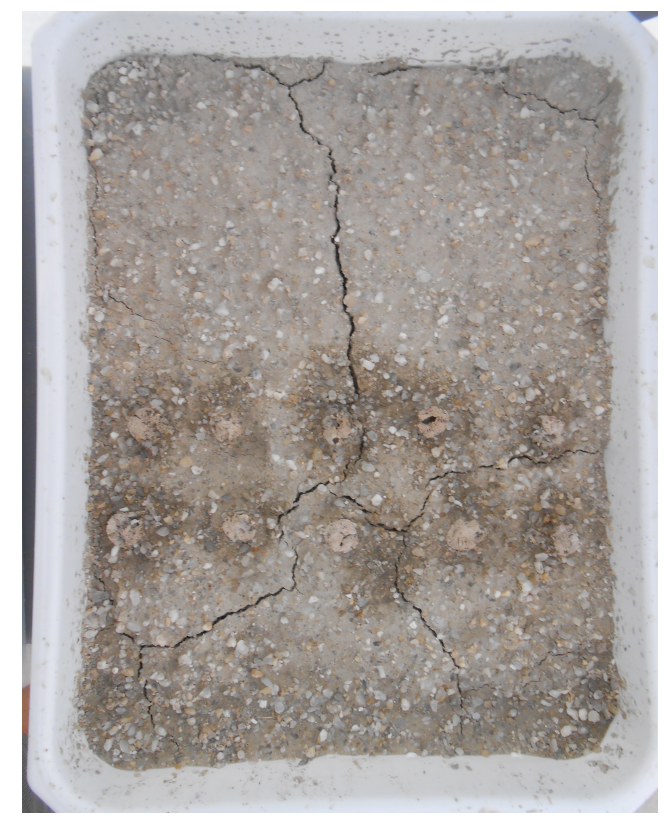

Los adhesivos se aplicaron directamente sobre la superficie (1 $\mathrm{mL}$ por talo), cubriendo un área de sustrato del mismo tamaño que el talo posibilitando así una correcta adhesión al sustrato. Una vez hecho esto se fueron colocando los diferentes talos de líquenes en su posición correspondiente. Para el posterior registro del posible movimiento se utilizó una cuadrícula metálica que permite la identificación de aquellos que pudieran presentar movimiento tras la simulación. Una vez preparadas las bateas se esperó una semana antes de comenzar la simulación de lluvia.

Simulación de lluvia

Para llevar a cabo la experiencia se utilizó un simulador de lluvia portátil de intensidad variable (Fernández-Gálvez et al., 2008). El simulador de lluvia (Foto 8) produce gotas de agua simulando la lluvia natural, está diseñado para usarse sobre una superficie de $50 \times 50 \mathrm{~cm}$. Consiste en una placa con 1250 goteros (Foto 7), los formadores de gotas son tubos capilares de plástico con diámetros internos y externos de 1 y $3 \mathrm{~mm}$, respectivamente, con un hilo de nylon insertado en el extremo con diámetro de $0.4 \mathrm{~mm}$, para aumentar la carga hidráulica y evitar la obstrucción por burbujas de aire. La placa formadora está sostenida por una estructura de metal a una cierta altura sobre la superficie del suelo. Ésta se encuentra conectada a un depósito de agua, la cual es 
suministrada por una bomba peristáltica regulable que mantiene la presión constante, controlada por un microprocesador.

Una vez que las bateas, con los talos convenientemente adheridos, estuvieron listas se procedió a calibrar la inclinación a la que las bandejas donde estaban los talos iban a recibir la precipitación. Se utilizó una inclinación del $40 \%$, lo suficientemente acentuada como para poder ocasionar escorrentía y por lo tanto movimiento en los talos. Esta pendiente estaba próxima a la máxima que soporta el material en la naturaleza, en caso de una mayor pendiente el sustrato es inestable (Salas, 2008). Siendo además un valor extremo puesto que las pendientes que podemos encontrar de forma natural en la zona de estudio no alcanzan, o rara vez lo hacen, este valor. Que tampoco se superará normalmente tras la explotación de la cantera según la memoria del estudio de impacto ambiental (Salas, 2008) y el plan de explotación de las canteras (KNAUF, 2008).

Por otro lado se decidió que la precipitación que se iba a aplicar en las simulaciones sería de $50 \mathrm{~L} / \mathrm{m}^{2} / \mathrm{h}$. Este valor simula un evento de lluvia muy intenso, lo suficiente para producir movimiento en los talos y además es un valor que representa un evento extremo de lluvia, que rara vez se produce en la zona de estudio (ver datos de pluviometría en Anexo 2).

Por último se decidió que el tiempo de duración de cada simulación sería de 15 minutos, ya que un evento de lluvia en la naturaleza de $50 \mathrm{~L} / \mathrm{m}^{2} / \mathrm{h}$ sostenido durante más de 10 ó 15 minutos son poco habituales en la zona de estudio (Ver datos de pluviometría en Anexo 2). Para poner a prueba los adhesivos se realizaron dos simulaciones por batea con una semana de separación entre ambas. De esta forma se podría ver como se comportan los adhesivos utilizados tras una precipitación intensa y como se verían afectados por la acción del agua y su posterior secado ante un nuevo evento de lluvia extremo.

El orden en el que se iban colocando las bateas en el simulador fue aleatorio. Al final de cada simulación, en cada talo se registró si permanecía adherido (0) o se había desplazado (1). 


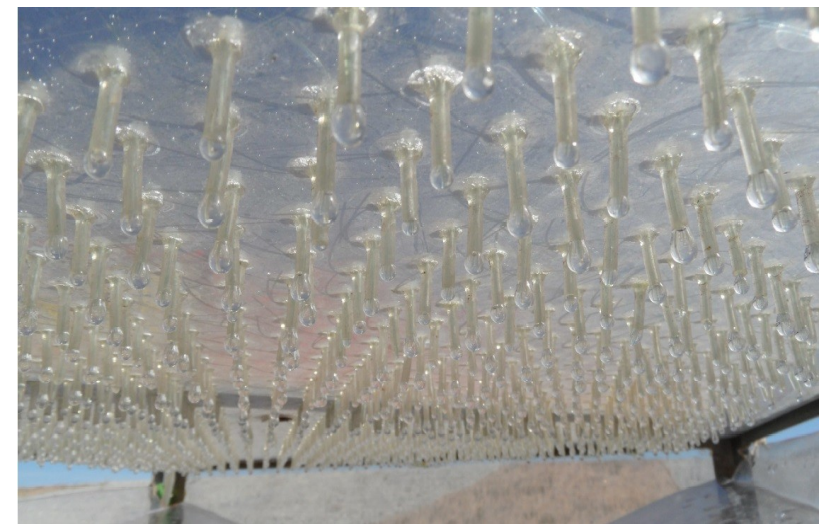

Foto 7. Detalle de los 1250 goteros que conforman la placa formadora. los formadores de gotas son tubos capilares de plástico con diámetros internos y externos de 1 y $3 \mathrm{~mm}$, respectivamente, con un hilo de nylon insertado en el extremo con diámetro de $0.4 \mathrm{~mm}$, para aumentar la carga hidráulica y evitar la obstrucción por burbujas de aire (Fotografía superior).

Foto 8. Simulador de lluvia con la descripción de todos los componentes que lo conforman incluyendo bomba peristáltica y depósito de agua. (Fotografía de la derecha).

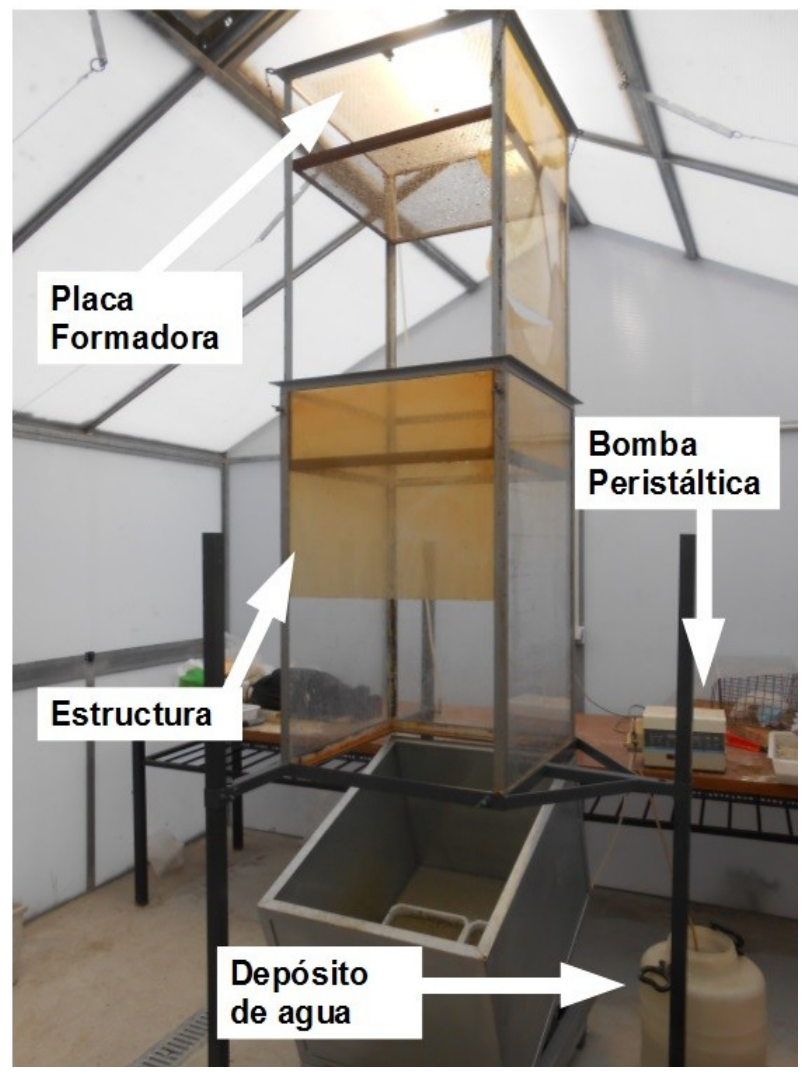

\section{Experiencia en campo}

El objetivo de esta experiencia fue comprobar la efectividad en campo de los adhesivos, descritos anteriormente, para mantener los talos liquénicos fijos al sustrato en una superficie plana (

La experiencia se llevó a cabo en la zona de estudio, concretamente en una de las áreas de acumulación de material de rechazo (subproducto de la cantera con un alto contenido en yeso). Este área fue previamente acondicionada por operarios de la empresa que explota la cantera (Knauf gmbh), (Foto 9). Este material fue seleccionado por haber mostrado los mejores resultados en experiencias de restauración de matorral gipsícola (Ballesteros et al., 2012, 2014). Además este sustrato, horizontal y no demasiado compactado, es similar al utilizado por la especie y la comunidad de la que forma parte (Acarosporetum placodiiformi-reagentis Llimona 1974) en condiciones naturales (Casares-Porcel \& Gutiérrez-Carretero, 1993). Sobre la superficie se delimitaron 30 parcelas de $0,25 \mathrm{~m}^{2}(50 \mathrm{x} 50 \mathrm{~cm})$ (Esquema 1). Correspondientes a los 6 
tratamientos (5 adhesivos más 1 control, ver detalles en apartado anterior; 5 réplicas x 6 tratamientos $=30$ réplicas). Los diferentes tratamientos de adhesivos fueron asignados a parcelas de forma aleatoria (Esquema 1).

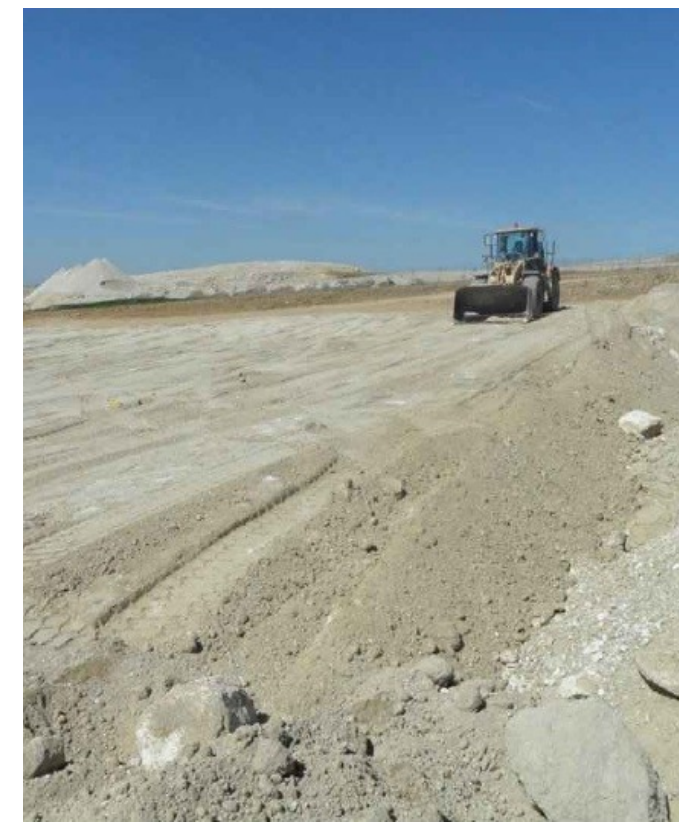

Foto 9. Acondicionamiento por parte de la empresa que explota las canteras de yeso de la parcela experimental.

\begin{tabular}{|c|c|c|c|}
\hline C5 & W3 & W2 & G1 \\
\hline G5 & HS3 & SR2 & AG1 \\
\hline AG5 & C3 & HS2 & SR1 \\
\hline SR5 & G4 & C2 & HS1 \\
\hline HS5 & AG4 & G3 & G2 \\
\hline C5 & SR4 & AG3 & C1 \\
\hline W5 & HS4 & SR3 & AG2 \\
\hline \multirow{2}{*}{ W } & W4 & & W1 \\
\hline
\end{tabular}

Esquema 1. Distribución aleatoria de las diferentes réplicas de los tratamientos en la zona habilitada para tal fin en el área de estudio. Control (C), Agua (W), Cola blanca (G), Pegamento de hidrosiembra (HS), Resina sintética (SR) y Goma arábiga (AG).

En cada parcela se colocaron un total de 35 talos de liquen con forma redonda y de un diámetro de 1,5 cm. El procedimiento para la obtención de talos fue el mismo que el descrito para la experiencia de laboratorio. En cada parcela se colocaron 35 siendo un total de 175 talos de liquen por tratamiento y un total de 1050 talos (Foto 12).

Para colocar los 35 talos de cada réplica de forma homogénea y sin variación entre las mismas se utilizó una cuadrícula metálica de muestreo de $0,5 \mathrm{~m}^{2}(0,5 \times 0,5 \mathrm{~m})$, dividido en 100 cuadrados de 5 x $5 \mathrm{~cm}$ (Foto 11). Se colocaron los 35 talos en el centro de cada parcela, en 7 filas de 5 líquenes cada una, en el centro de los cada cuadrado de 5 x $5 \mathrm{~cm}$., siendo la separación entre los talos de $5 \mathrm{~cm}$. dentro de cada parcela. Para aplicar los diferentes adhesivos se utilizaron jeringas de 
plástico para ser lo más precisos posibles en la cantidad de adhesivo aplicado por talo ( $1 \mathrm{~mL}$ por talo) (Foto 10).

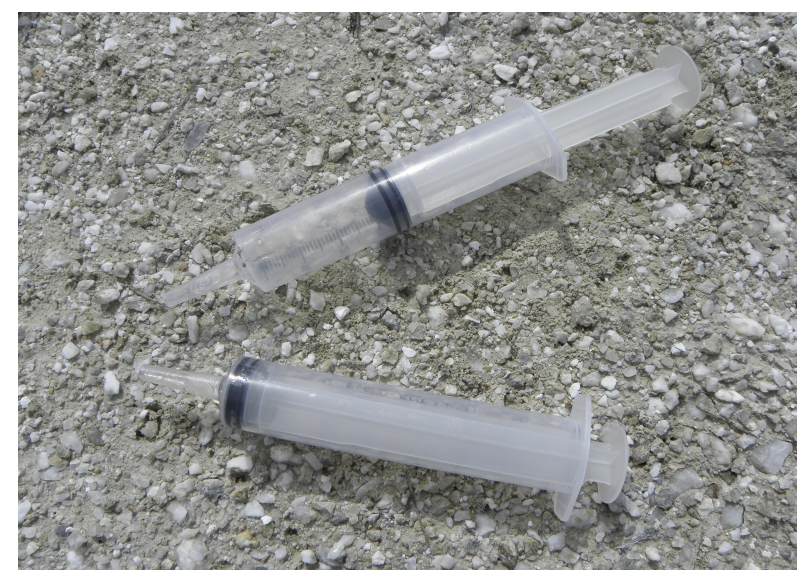

Foto 10. Jeringas de plástico que se utilizaron para aplicar de forma precisa los diferentes adhesivos al sustrato (Arriba).

Foto 11. Para colocar los líquenes en sus respectivas parcelas se utilizó una cuadrícula metálica con 100 cuadrículas de 5 x $5 \mathrm{~cm}$. (A la derecha)

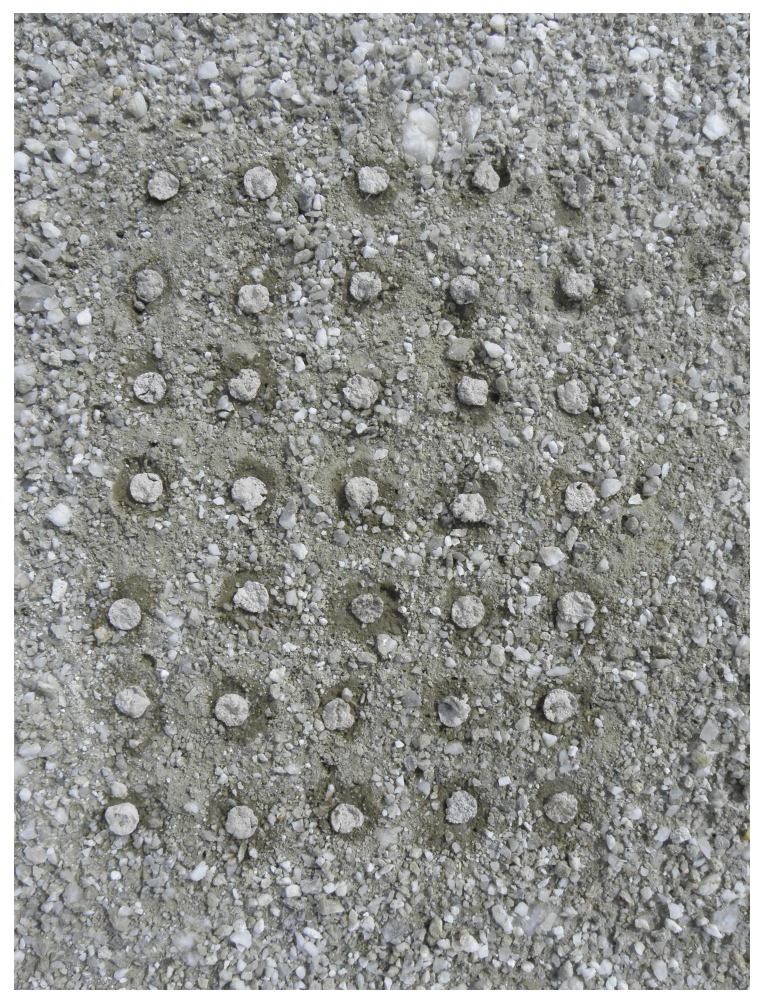

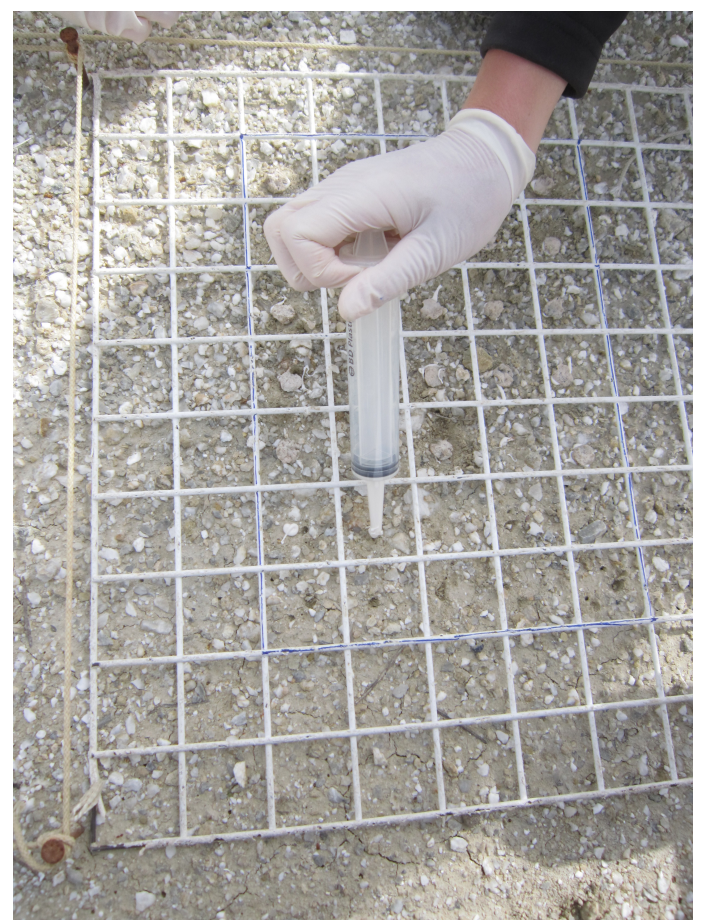

Foto 12. Resultado final de la colocación de los talos una vez aplicado el correspondiente adhesivo, cada réplica contenía 35 talos dispuestos en 7 filas con 5 talos cada una. (Abajo). 
La fecha seleccionada para montar la experiencia en campo fue el 21 de marzo. Se eligió la primavera por dos razones: 1) La climatología variable de esta época, con eventos de precipitación de intensidad variable, a veces torrenciales, registrándose con frecuencia vientos de fuerte intensidad y 2) Las restauraciones suelen realizarse en su mayoría en esta estación. Se esperó una semana para que los adhesivos hicieran su efecto, a partir de este momento se comenzó a registrar los talos que se habían desplazado, siendo entonces cuando se comenzó a tomar medidas del movimiento de los talos.

El registro del movimiento de los talos en los diferentes tratamientos se llevó acabo de forma semanal, hasta la semana 12 y mensual a partir de esta semana. En esta memoria se presentan los datos de los 14 primeros muestreos. En cada muestreo se anotaba el número de talos adheridos (1) frente al número de talos desplazados (0) en cada parcela. Además los talos que se iban moviendo fueron retirados con el objetivo de que no interfirieran en posteriores registros de movimiento.

\section{Viabilidad de los Líquenes}

Otro aspecto relevante es conocer si los adhesivos afectan a la funcionalidad biológica de los líquenes. La evaluación de la actividad de la costra biológica del suelo puede realizarse por varios métodos, aunque tradicionalmente el más utilizado (Lange, 2003; Lange \& Green, 2006; CastilloMonroy et al., 2011; Bowling et al., 2011) es la medición del intercambio de $\mathrm{CO}_{2}$, que permite estimar tanto la actividad catabólica (emisiones de $\mathrm{CO}_{2}$ ) como la fotosintética (absorción de $\mathrm{CO}_{2}$ ). En nuestro caso hemos utilizado una cámara de respiración SRC-1, junto con un monitor de gas ambiental EGM-4 (PP- Systems Inc.), estos instrumentos son habitualmente utilizados en este tipo de estudios (Jensen et al., 1996) por ser asequibles y funcionales, debido a su fácil transporte y fiabilidad (Foto 13). 


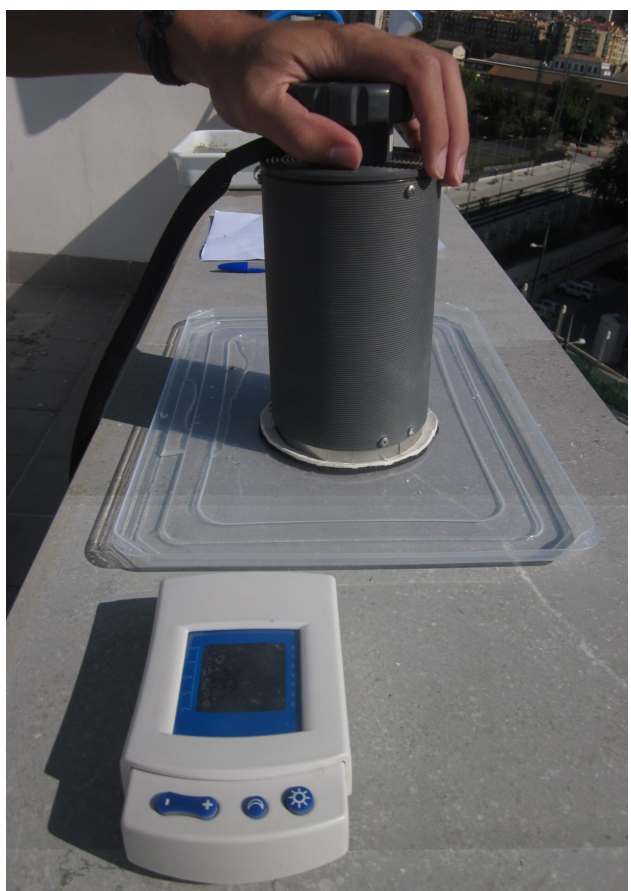

Foto 13. Proccedimiento de toma de los valores de respiración de los talos que habían sido expuestos a los diferentes tratamientos. Los valores se tomaron con una cámara de respiración tomando además medidas de la temperatura ambiental para realizar un correcto calibrado del dispositivo

De forma preliminar al experimento, se hizo una prueba con distinto número de talos, tras la cual se comprobó que se obtenían datos contrastados a partir de tres talos liquénicos. Finalmente se incluyeron 6 talos en cada medición (procedentes de una misma batea y con el mismo adhesivo). En total, se hicieron 5 medidas de respiración por tratamiento (correspondientes a cada una de las bateas utilizadas en la experiencia de laboratorio). También se realizaron 5 medidas sin talos con el objetivo de establecer un control del propio aparato.

\section{Análisis estadístico}

El efecto de los adhesivos en el desplazamiento de los talos en campo y laboratorio se analizó ajustando Modelos Lineales Generalizados (GLMs), especificando un error de tipo binomial y la función de vínculo "logit" empleando el paquete de R "stats" (R Development Core Team, 2014). El movimiento de los talos en el campo se representó mediante curvas de supervivencia de Kaplan-Meier con el paquete de R "survival” (Therneau, 2013). Mientras que el efecto de los tratamientos en la respiración de los talos se evaluó mediante un GLM, especificando un error de tipo Poisson y la función de vínculo "log" usando también el paquete de R "stats". Las 
comparaciones múltiples realizadas en los análisis se llevaron a cabo empleando el paquete de $\mathrm{R}$ "multcomp" (Hothorn et al., 2008).

\section{RESULTADOS}

\section{Experiencia de laboratorio}

Después de haber realizado sendas simulaciones de lluvia el movimiento total que presentaron los talos de los diferentes tratamientos fue inferior al 2,3\%. El desplazamiento se concentró en los talos control (con un $12 \%$ de talos desplazados) a los que no se les aplicó ningún tipo de adhesivo. (Tabla 1).

\begin{tabular}{|c|c|c|c|}
\hline \multirow{2}{*}{ Tratamiento aplicado } & \multicolumn{2}{|c|}{$\mathbf{N}^{0}$ de Pseudoréplicas desplazadas } & \multirow{2}{*}{ TOTAL } \\
\cline { 2 - 4 } & $1^{\text {a }}$ Simulación & $2^{\text {a }}$ Simulación & \\
\hline Goma arábiga & 0 & 0 & 0 \\
\hline Resina sintética & 0 & 0 & 0 \\
\hline Cola blanca & 0 & 0 & 0 \\
\hline Gel hidrosiembra & 1 & 0 & $\mathbf{1}$ \\
\hline Agua & 0 & 0 & 0 \\
\hline Control & 6 & 0 & $\mathbf{6}$ \\
\hline \multicolumn{2}{|c|}{} & & 7 \\
\cline { 3 - 4 } & & &
\end{tabular}

Tabla 1. Número de talos desplazados en cada tratamiento tras dos simulaciones de lluvia.. Todo el movimiento de los talos se registró durante la primera simulación. 
Foto 14. Resultado tras la simulación de lluvia n una batea con talos de liquen control. Se observan los talos desplazados por la acción del agua. (Derecha)

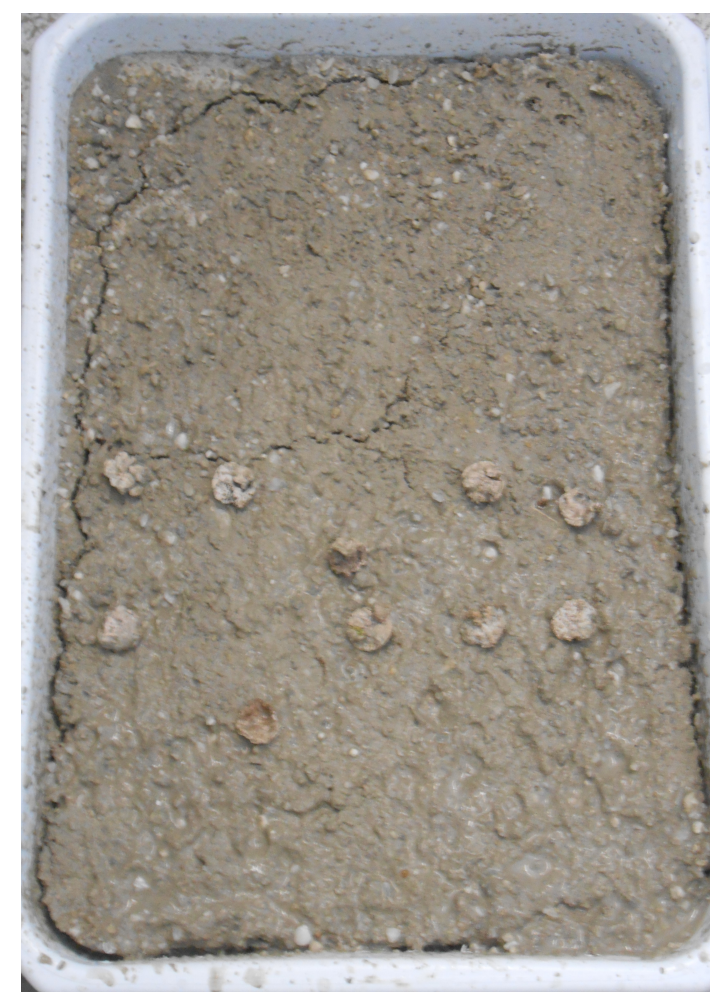

El movimiento se registró únicamente tras la primera simulación de lluvia (Fotos 14 y 15). No hubo desplazamiento de ningún talo durante el desarrollo de la segunda simulación. (Gráfica 1). Cabe destacar que los talos tratados con resina sintética (SR) como adhesivo sufrieron un progresivo oscurecimiento debido, probablemente, a que dicho adhesivo afectó a la viabilidad de los líquenes (ver apartado de la viabilidad de los líquenes).

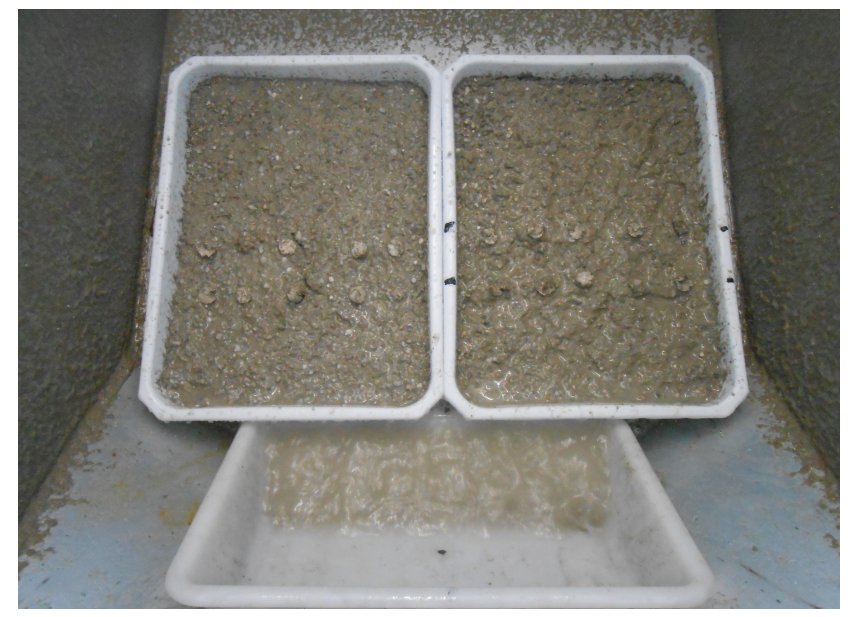

Foto 15. En la imagen se observan dos de las bateas correspondientes a dos tratamientos diferentes que están siendo expuestas a la acción del simulador de lluvia. 


\section{Movimiento simulacion 1}

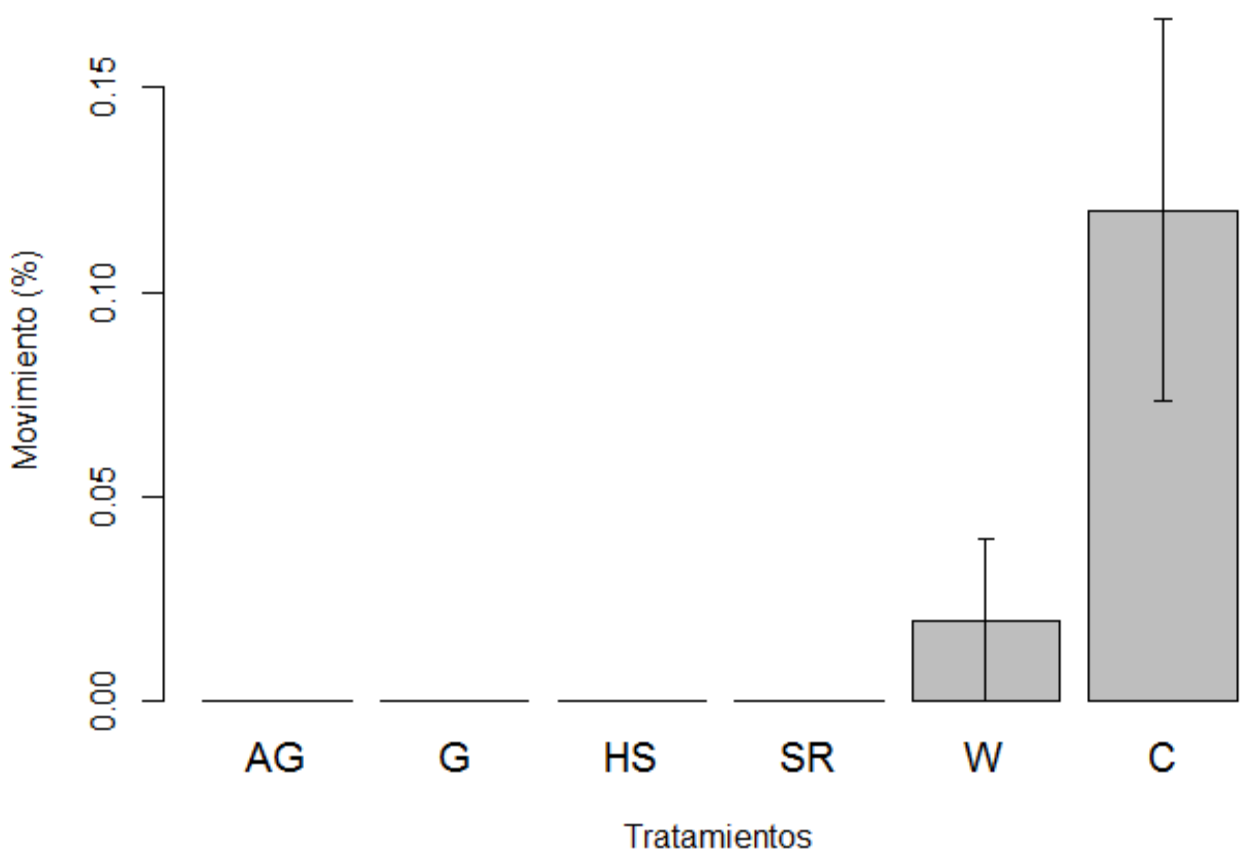

Gráfica 1. Porcentaje de talos por tratamiento que se habían desplazado después de realizar la primera simulación. En la parte de abajo semuestran los difeerentes tratamientos, Control (C), Agua (W), Cola blanca (G), Pegamento de hidrosiembra (HS), Resina sintética (SR) y Goma arábiga (AG).

No se presentaron diferencias significativas entre los diferentes tratamientos en cuanto al porcentaje de talos desplazados. (Tabla 2)

\begin{tabular}{lcccc}
\hline Movement & Estimate & SE & z value & $\operatorname{Pr}(>|\mathrm{z}|)$ \\
\hline Intercept & $-2.157 \mathrm{e}+01$ & $4.134 \mathrm{e}+03$ & -0.005 & 0.996 \\
$\mathrm{G}$ & $5.146 \mathrm{e}-10$ & $5.846 \mathrm{e}+03$ & 0.000 & 1.000 \\
$\mathrm{HS}$ & $5.149 \mathrm{e}-10$ & $5.846 \mathrm{e}+03$ & 0.000 & 1.000 \\
$\mathrm{SR}$ & $5.145 \mathrm{e}-10$ & $5.846 \mathrm{e}+03$ & 0.000 & 1.000 \\
$\mathrm{~W}$ & $1.767 \mathrm{e}+01$ & $4.134 \mathrm{e}+03$ & 0.004 & 0.997 \\
$\mathrm{C}$ & $1.957 \mathrm{e}+01$ & $4.134 \mathrm{e}+03$ & 0.005 & 0.996 \\
\hline
\end{tabular}

Tabla 2. Efecto de los tratamientos con diferentes adhesivos sobre el movimiento de los talos evaluado mediante un Modelo Lineal Generalizado (GLM). Control (C), Agua (W), Cola blanca (G), Pegamento de hidrosiembra (HS), Resina sintética (SR) y Goma arábiga (AG). 


\section{Experiencia de campo}

En cuanto a la experiencia de campo se observó, en los primeros controles de movimiento, un desplazamiento de gran número de los talos que no fueron sometidos a ningún tratamiento (Control). Conforme fueron pasando las semanas se observó como los talos en los que se usó agua (W) y cola blanca (G) como adhesivo presentaron un menor movimiento (en torno al 5\% en el caso de la cola blanca y al $10 \%$ en el caso del agua), sin embargo, se registró que los talos que fueron adheridos al sustrato con goma arábiga (AG), pegamento de hidrosiembra (HS) y resina sintética (SR), presentaban un mayor desplazamiento en cada uno de los registros que se iban efectuando, aunque no llegando nunca a los niveles de movimiento que se registraron en el tratamiento control, cuyo número de talos desplazados es el mayor de todos los tratamientos de la experiencia (un $50 \%$ del total). (Gráfica 2). Cabe decir que los talos expuestos al adhesivo resina sintética tomaron un color oscuro al igual que ocurrió en la experiencia de laboratorio.

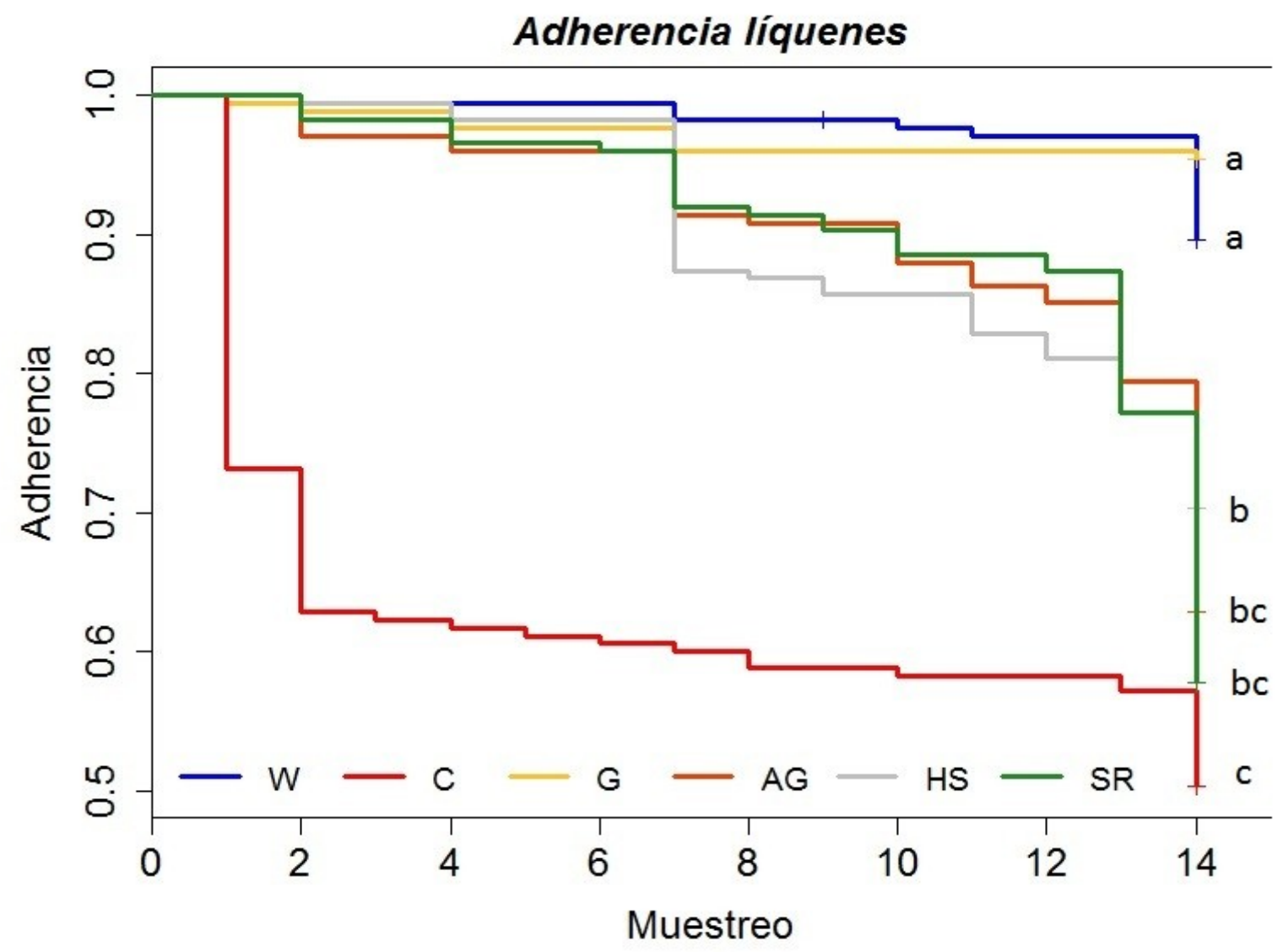

Gráfica 2. Desplazamiento de los líquenes representado mediante curvas de Kaplan-Meier. Los doce primeros valores corresponden a seguimientos semanales y los dos últimos al desplazamiento registrado al final de los meses de Julio y Agosto. Diferentes letras al final de las curvas indican diferencias significativas entre tratamientos $(\mathrm{p}<0.05)$. Los diferentes tratamientos son, Control (C), Agua (W), Cola blanca (G), Pegamento de hidrosiembra (HS), Resina sintética (SR) y Goma arábiga (AG). 
Encontramos tres grupos homogéneos entre los cuales no se dan diferencias significativas. En primer lugar encontramos a los tratamientos cola blanca $(G)$ y agua (W) en los que se desplazaron menos talos (Un $5 \%$ y un $10 \%$ respectivamente), a continuación están los tratamientos con Pegamento de hidrosiembra (HS) por un lado y resina sintética (SR) y goma arábiga (AG) por otro, donde el movimiento presentado por los talos fue mayor y por último el grupo del tratamiento control (C) que como hemos comentado anteriormente fue el que menor efectividad presentó a la hora de fijar los talos al sustrato. (Ver tabla 3).

\begin{tabular}{lcccc}
\hline Movement & Estimate & SE & z value & Pr $(>|\mathbf{z}|)$ \\
\hline Intercept & -2.1659 & 0.2488 & -8.704 & $<0.0001$ \\
G & -0.8727 & 0.4392 & -1.987 & $<0.0001$ \\
C & 2.1544 & 0.2912 & 7.399 & $<0.0001$ \\
AG & 1.6398 & 0.2939 & 5.579 & $<0.0001$ \\
HS & 1.3049 & 0.2988 & 4.367 & $<0.0001$ \\
SR & 1.8548 & 0.2921 & 6.349 & $<0.0001$ \\
\hline
\end{tabular}

Tabla 3. Efecto de los tratamientos con diferentes adhesivos sobre el movimiento de los talos evaluado mediante un Modelo Lineal Generalizado (GLM). Control (C), Agua (W), Cola blanca (G), Pegamento de hidrosiembra (HS), Resina sintética (SR) y Goma arábiga (AG).

\section{Viabilidad de los líquenes}

En cuanto a la viabilidad de los líquenes, se observaron diferencias significativas entre los valores de emisión de $\mathrm{CO}_{2}$ de cada tratamiento. El tratamiento control (C) registró los valores más elevados, seguidos por el pegamento de hidrosiembra (HS) y el agua (W), mientras que los correspondientes a los tratamientos con goma arábiga $(\mathrm{AG})$, cola blanca $(\mathrm{G})$ y resina sintética $(\mathrm{SR})$ presentaron una respiración menor. El control realizado empleando la cámara vacía $(\mathrm{CC}$, sin ningún talo) arrojó los valores más bajos de respiración (Gráfica 3). 


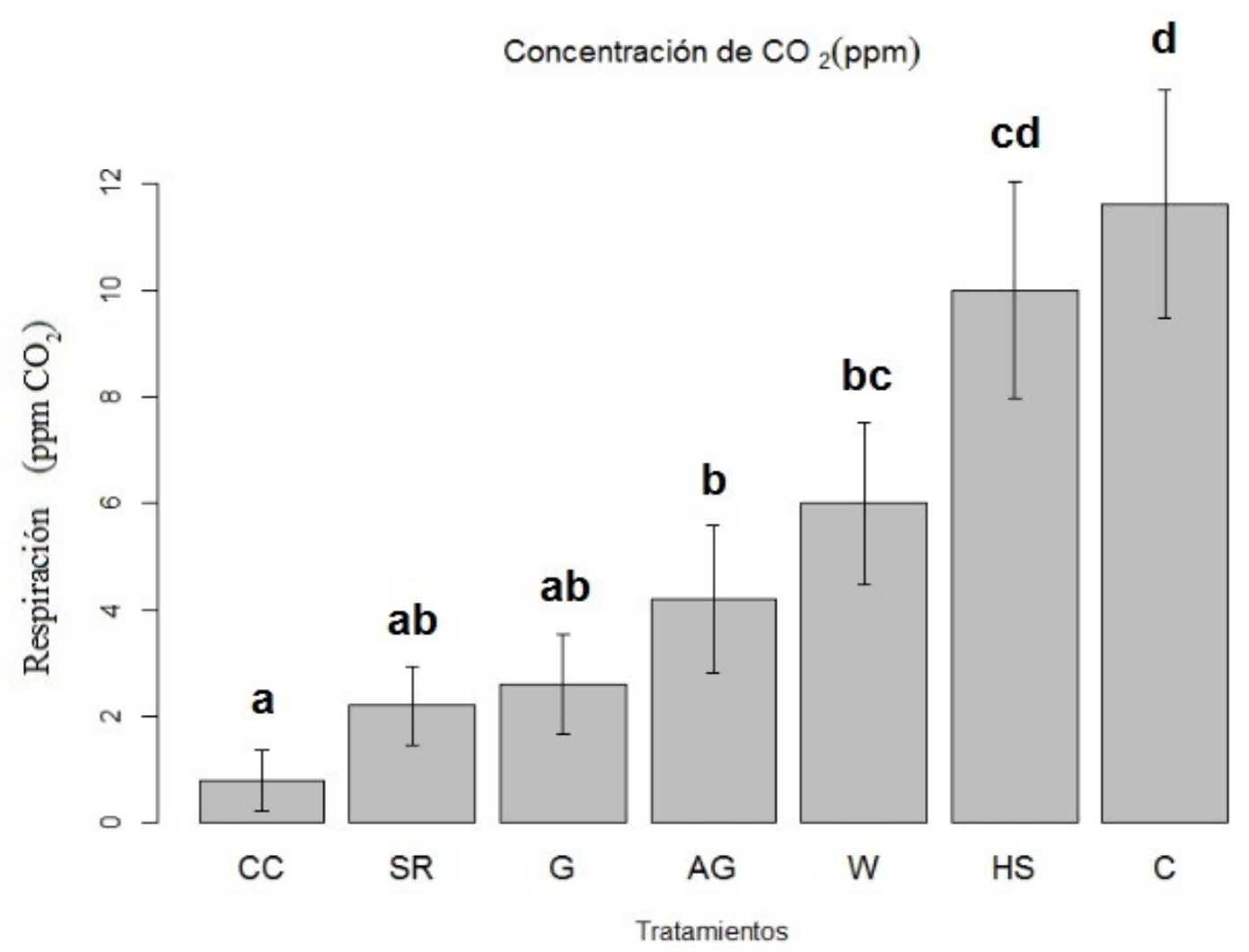

Gráfica 3. Se observa la respiración generada por los talos según el tratamiento de adhesivos al que fueron expuestos $\left(\mathrm{ppm} \mathrm{CO}_{2}\right)$. El mayor registro correspondió a los talos Control, mientras que el menor correspondió a las medidas que se realizaron con la cámara vacía (CC). Las letras que aparecen en la parte superior de cada barra corresponden a las diferencias significativas entre los diferentes tratamientos. Abajo se muestran los diferentes tratamientos: Control (C), Agua (W), Cola blanca (G), Pegamento de hidrosiembra (HS), Resina sintética (SR) y Goma arábiga (AG).

No hubo diferencias significativas entre el tratamiento control (C) y el Pegamento de hidrosiembra (HS). No se encontraron diferencias significativas entre el tratamiento con Pegamento de hidrosiembra (HS) y el tratamiento con agua (W). Un grupo de tratamientos sin diferencias significativas entre ellos fue, el agua (W), la goma arábiga (AG), la cola blanca (G) y la resina sintética (SR). Por último no se presentaron diferencias entre otro grupo de tratamientos, el control de la cámara (CC), resina sintética (SR) y la cola blanca $(\mathrm{G})$, (Tabla 4). 


\begin{tabular}{lcccc}
\hline Movement & Estimate & SE & z value & $\operatorname{Pr}(>|z|)$ \\
\hline Intercept $(\mathrm{CC})$ & -0.2231 & 0.4999 & -0.446 & 0.655343 \\
$\mathrm{SR}$ & 1.0116 & 0.5838 & 1.733 & 0.083140 \\
$\mathrm{G}$ & 1.1787 & 0.5717 & 2.062 & 0.039242 \\
$\mathrm{AG}$ & 1.6582 & 0.5455 & 3.040 & 0.002366 \\
$\mathrm{~W}$ & 2.0149 & 0.5322 & 3.786 & $<0.0001$ \\
$\mathrm{HS}$ & 2.5257 & 0.5195 & 4.861 & $<0.0001$ \\
$\mathrm{C}$ & 2.6741 & 0.5169 & 5.174 & $<0.0001$ \\
\hline
\end{tabular}

Tabla 4. Efecto de los tratamientos con diferentes adhesivos sobre la respiración de los talos evaluado mediante un Modelo Lineal Generalizado (GLM). En la primera columna encontramos los diferentes tratamientos: Control (C), Agua (W), Cola blanca (G), Pegamento de hidrosiembra (HS), Resina sintética (SR) y Goma arábiga (AG).

\section{DISCUSIÓN}

\section{Experiencia de laboratorio}

El reducido desplazamiento en los talos, registrado en la experiencia de simulación de lluvia bajo condiciones adversas (precipitación y pendiente elevadas), mostró el efecto limitado de la lluvia combinada con la pendiente sobre el desplazamiento de los talos. Tan solo se desplazaron los talos en los tratamientos control, aunque tras el primer evento de lluvia, incluso estos talos sin adhesivo quedan fijados al sustrato de forma efectiva, no registrándose movimiento en el segundo evento, este es el proceso de dispersión y fijación que ocurre en condiciones naturales (Nash, 1996). Los resultados además justificaron la experiencia de campo, donde entraba en juego el viento, factor muy importante en la costras de zonas áridas (Moore, 1998).

El resultado de la simulación arroja una idea clara y es que los talos que no se fijan al sustrato con la ayuda de algún adhesivo presentan una mayor probabilidad de desplazarse con respecto a su posición inicial. Si extrapolamos esto a una hipotética restauración de la costra liquénica en campo, supondría que los talos que se desplacen no tendrían el tiempo suficiente para 
que el líquen se fije al sustrato de forma natural, y aumentaría el riesgo de que el talo se desplace fuera de los afloramientos de yesos. De hecho es sabido por anteriores estudios (Belnap et al., 1998) que el tiempo de asentamiento y posterior regeneración de las costras biológicas es muy prolongado. Por lo tanto, el no aplicar ningún adhesivo es una opción descartable desde el punto de vista de la lluvia y las pendientes fuertes.

Cabe destacar que el movimiento ocasionado sobre los talos que no se fijaron mediante el uso de adhesivo alguno se registró en la primera de las simulaciones no produciéndose movimiento en la segunda. Este aspecto es importante ya que tras la primera simulación se esperó el tiempo necesario para que el sustrato se secara completamente antes de llevar a cabo la segunda, ocasionando esto que el agua al mezclarse con el sustrato rico en yeso actuara como cementante una vez se hubo secado el mismo. Esto supone un punto a favor de la utilización de agua como método para fijar los talos al sustrato. Dicho de otra forma el tratamiento control (sin adhesivo) deja de serlo a efectos prácticos una vez que este entra en contacto con el agua.

\section{Experiencia de campo}

En campo las diferencias entre los tratamientos en los que se utilizó adhesivo y el control han sido bastante evidentes, apareciendo diferencias estadísticamente significativas en muchos casos. El desplazamiento de los talos en el tratamiento control fue drástico en las dos primeras semanas (superior al 35\%), suavizándose en los siguientes muestreos, pero diferenciándose del resto de tratamientos en los que el movimiento fue menor, más gradual y con menores diferencias. El viento, factor clave en este experimento, se mostró como factor decisivo en el desplazamiento de los talos en las dos primeras semanas en las que además no se registró ninguna precipitación. La disminución paulatina del movimiento de los talos que no se fijaron con adhesivo (C), puede deberse a la acción que el agua de lluvia produce, ya que como se observó en la experiencia llevada a cabo en el laboratorio, el agua al interaccionar con el sustrato actúa como cementante ocasionando que los talos de liquen queden adheridos.

Los tratamientos adhesivos más efectivos fueron la cola blanca $(\mathrm{G})$ y el agua (W) con un porcentaje de adherencia superior al 90\%, seguidos del pegamento de hidrosiembra (HS) próximo 
al 70\% y el resto de tratamientos con porcentaje inferior al 63\%. Si comparamos la efectividad entre los diferentes tratamientos con adhesivos se observa que la cola blanca (G) y el agua (W) son los más efectivos. Se observó que humedecer la superficie antes de colocar los talos (W) era el tratamiento que mejor conseguía incluir al talo en el sustrato. Cabe destacar que es el tratamiento más fácil de aplicar y por supuesto el más barato. El agua es además inocua y rehidrata el talo favoreciendo la actividad del fotobionte y del micobionte (Smith, 1973; Brock, 1975) sobre todo durante las primeras horas del día (Lange, 2003, 2007; Lange et Green, 2006).

Es preciso comentar que en el tratamiento con cola blanca $(\mathrm{G})$ el adhesivo, al endurecerse, forma una capa que aísla el talo con respecto al sustrato impidiendo un correcto desarrollo de las hifas lo cual puede resultar perjudicial para un normal desarrollo del líquen.

En cuanto al resto de los adhesivos, vemos inadecuado el uso de la resina sintética (SR), con un elevado número de talos desplazados y cambios en color y textura (ver apartado de viabilidad de los líquenes más abajo). La goma arábiga (AG) también presentó al final de la experiencia de campo muchos talos desplazados, aunque no se apreciaron cambios en la textura y/o color de los mismos. Cabe destacar para el resto de tratamientos (AG y SR) que su baja efectividad a la hora de adherir los líquenes al sustrato puede deberse a que estos tratamientos forman una costra dura por debajo de los talos que impide el desarrollo de las hifas desde la parte inferior del mismo.

Como sabemos, una de las propiedades mejor conocidas de las costras biológicas es su lenta regeneración tras una perturbación (Bowker et al. 2007). Algunos ejemplos en la literatura han estimado tiempos de recuperación de 6 años tras perturbaciones moderadas (e.g. pisoteo, Belnap \& Eldridge 2003) hasta siglos (Eldridge \& Ferris 1999) o milenios (Belnap \& Warren 1998) ante perturbaciones más drásticas (Bowker et al. 2007). Es por esto, que el hecho de encontrar adhesivos que prolonguen la estabilidad de los líquenes tras una traslocación, como ha sido en nuestro caso el agua (W) o el pegamento de hidrosiembra (HS) sea tan relevante, ya que hace posible abordar con más garantías una posible restauración.

Como síntesis de este apartado, es relevante destacar que para contrarrestar el efecto del viento, factor fundamental en la dispersión y la biología de los líquenes (Heinken, 1999), pero que puede provocar el fracaso de una actuación como esta, es necesario aplicar algún tipo de adhesivo 
que afecte lo menos posible a la funcionalidad del liquen y prolongue su estabilidad en el sustrato durante un periodo de tiempo suficiente para posibilitar su estabilidad dentro del sustrato mediante el desarrollo de mecanismos naturales.

\section{Viabilidad de los líquenes}

Como ya comentamos en el apartado de resultados, hubo diferencias significativas entre los tratamientos en cuanto a la tasa de respiración. Entre los tratamientos que presentaron una mayor tasa de respiración encontramos el pegamento de hidrosiembra (HS) y el agua (W). En el resto de tratamientos (AG, G y SR) se registró una tasa de respiración significativamente más baja.

Dichos resultados son lógicos desde el punto de vista de la composición de los adhesivos, ya que el agua es inocua y el Pegamento de hidrosiembra (HS) es una sustancia tradicionalmente utilizada en labores de restauración (Matesanz et al., 2006; Tormo et al., 2007) no presentando toxicidad para los organismos vegetales, tampoco produce efecto alguno sobre su funcionalidad biológica. Por el contrario el resto de adhesivos (AG, G y SR) por un lado, pueden contener sustancias químicas que presumiblemente afectarían de alguna forma a la funcionalidad biológica de los líquenes produciendo un descenso en la tasa de respiración con respecto a los tratamientos mencionados en el párrafo anterior y por otro lado parece que de alguna forma aíslan al líquen del sustrato.

Por lo tanto los tratamientos con agua (W) y Pegamento de hidrosiembra (HS) se perfilan como los más adecuados para su uso como adhesivos en una hipotética restauración, ya que, además de no presentar variaciones con respecto al control en cuanto a la tasa de respiración después de su aplicación, presentaron bajas tasas de movimiento en la experiencia de campo (ver apartado anterior). 


\section{CONCLUSIONES}

Tras haber realizado las experiencias en laboratorio y campo, y habiendo analizado los resultados junto con la bibliografía correspondiente, hemos obtenido las siguientes conclusiones:

- El viento es el principal agente dispersante en el caso de talos de Diploschistes diacapsis en el área de estudio y probablemente podemos asumir que también lo sea en gran parte de especies de líquenes de la costra liquénica que encontramos en esta zona.

- El agua en forma de precipitaciones también resulta un factor importante en la dispersión de los talos pero parece ser menos determinante que el viento.

- Debido al lento crecimiento que presentan los líquenes se debe valorar en todo momento la traslocación como método de introducción de la costra liquénica en la zona que se pretenda restaurar.

- La traslocación de talos de liquen agiliza y acelera el proceso de restauración de la costra liquénica.

- Los adhesivos más adecuados en una hipotética restauración serían el agua (W) por su bajo coste y fácil aplicación y el Pegamento de hidrosiembra (HS) por ser una sustancia comúnmente utilizada en labores de restauración y de fácil aplicación.

- El uso de agua como adhesivo en una hipotética restauración podría ser extrapolable, al menos, a aquellas zonas donde la composición predominante del suelo sea, como en nuestra experiencia, el yeso. 


\section{BIBLIOGRAFÍA}

AgUIlar J., SIERRA C., ORTEgA E., ROCA A., SAURA I., ASENSIO C. 1992. Mapa de suelos 1:100.000 Padul-1026. ICONA. Ministerio de Agricultura, Pesca y Alimentación, Madrid.

ALDAYA F., VERA J.A., FONTBOTE J.M. 1980. Mapa geológico de España. E. 1:200.000. Granada-Málaga. IGME. Servicio de Publicaciones. Ministerio de Industria y Energía.

ANÓNIMO. 1992. Council Directive 92/43/EEC on the conservation of natural habitats and of wild fauna and flora. European Commission's Directorate-General for the Environment. Brussels.

\section{BALleSteros M., CAÑAdAS E. M., FORONDA A., FERNÁNDEZ-ONDOÑO E., PEÑAS} J., LORITE J. 2012. Vegetation recovery of gypsum quarries: short-term sowing response to different treatments. Applied Vegetation Science 15: 187-197.

BAllesteros M., CAÑAdas, E.M., FORONdA A., PEÑAS J., VALLE F., LORITE J., 2014. Central role of bedding materials for gypsum-quarry restoration: An experimental planting of gypsophile species. Ecological Engineering, 70: 470-476.

BAÑARES Á., BLANCA G., GÜEMES J., MORENO J.C., ORTIZ S. 2004. Atlas y Libro Rojo de la Flora Vascular Amenazada de España. Dirección General de Conservación de la Naturaleza. Madrid.

BARRENO E., MERINO A. 1981. Catálogo liquénico de las calizas de Madrid (España). Lazaroa 3: $247-268$.

BATES D., MAECHLER M., BOLKER B., WALKER S., 2013. Ime4: Linear mixed-effects Models Using S4 Classes. R Package Version 1.0-5.

BELNAP J., GILLETTE D.A. 1997. Disturbance of biological soil crusts: impacts on potential wind erodibility of sandy desert soils in southeastern Utah. Land degradation \& Development 8: 355-362. 
BELNAP J., GILLETTE D.A. 1998. Vulnerability of desert biological soil crusts to wind erosion: the infuences of crust development, soil texture, and disturbance. Journal of Arid Environments 39: 133-142.

BELNAP J., WARREN S. 1998. Measuring restoration success: a lesson from Patton's tank. Ecol Bull. $76: 33$

BELNAP J., ELDRIDGE D. 1993. Disturbance an recovery of biological soil crusts. Ecological studies. 150:363-383.

BENEDICT J. B. 1967. Recent glacial history of an alpine area in the Colorado Front Range, USA. I. Establishing a lichen-growth curve. Journal Glaciology. 6: 817-832.

BOYADGIEV T. G., VERHEYE W. H. 1996. Contribution to a utilitarian classification of gypsiferous soil. Geoderma 74: 321-338.

BOWKER M., BELNAP J., DAVIDSON D.W., PHILLIPS S.L. 2005. Evidence for micronutrient limitation of biological soil crusts: importance to arid-lands restoration. Ecological Applicatons 15: 1941-1951.

BOWKER M.A. 2007. Biological soil crust rehabilitation in theory and practice: an underexploited opportunity. Restoration Ecology 15: 13-23.

BOWKER M., BELNAP J. 2008. A simple clasification of soil types as habitats of biological soil crusts on the Colorado Plateau, USA. Journal of Vegetation Science 19: 831-840.

BOWLING D.R., GROTE E.E., BELNAP J. 2011. Rain pulse response of soil $\mathrm{CO}_{2}$ exchange by biological soil crusts and grasslans of the semiarid Colorado Plateau, United States. Journal of Geophysical Research 116. 
BROCK T.D. 1975. The effect of water potential on photosynthesis in whole lichens and in their liberated algal components. Planta. 124:13-23.

BURGAZ A.R., SERIÑA E. 1986. Contribución al catálogo de líquenes. Trabajos del Departamento del Botánica 13: 15-18.

CABLE J.M., HUXMAN T.E. 2004. Precipitation pulse size effects on Sonoran Desert soil microbial crusts. Oecologia 141: 317-324

CASARES-PORCEL M., GUTIÉRREZ-CARRETERO L. 1993. Síntesis de la vegetación liquénica gipsícola termo- y mesomediteránea de la Península ibérica. Cryptogamie Bryologie Lichenologie 14: 361-388.

CASARES-PORCEL M., GONZALES-TEJERO M., BOUCHAALAH M. 1994. Contribution to the knowledge of the gypsicolous lichen flora of Morocco. Cryptogamie-Bryologie et Lichenologie. 15:239-244.

CASARES-PORCEL M., HAFELLNER J., GUTIERREZ-CARRETERO L. 1996. Species of the genus Lecidea (Lecnorales) on gypsum in Spain. Lichenologist 28: 37-47.

CASARES-PORCEL M., GUTIÉRREZ-CARRETERO L. 2004. Los líquenes de las zonas subdesérticas de Tabernas. En J. F. Mota et al.. Subdesiertos de Almería: naturaleza de cine: 110121. Consejería de Medio Ambiente Junta de Andalucía, Sevilla.

CASTILLEJO J.M. \& CASTELLÓ R. 2010. Influence of the application rate of an organic amendment (Municipal Solid Waste) on gypsum quarry rehabilitation in semiarid environments. Arid Land Research and Management 24: 344-364.

CASTILlO-MONROY A.P., MAESTRE F.T., REY A., SOLIVERES S., GARCÍAPALACIOS P. 2011. Biological soil crust microsites are the main contributor to soil respiration in a semiarid ecosystem. Ecosystems 14: 835-847. 
CRESPO A., BARRENO E., RICO V.J., BUENO A.G. 1980. Catálogo liquénico del desierto de Calanda (Teruel, España). Anales del Jardín Botánico de Madrid.

DE BOODT M. 1993. Soil conditioning, a modern procedure for restoring physical soil degradation. Pedologie. 43:157-195.

DEINES L., ROSENTRETER R., ELDRIDGE D.J., SERPE M.D. 2007. Germination and seeding establishment of two annual grasses onlichen-dominated biological soil crusts. Plant Soil 295: 23-35.

DRIESSEN P.M., DUDAL R. 1991. The major soils of the world. Lecture notes on their geography, formation, properties and use. Agricultural University Wageningen- Katholieke Univ. Leuvev. Netherlands.

EGEA J.M., ALONSO F.L. 1996. Patrones de distribución en la flora liquénica xerófila del sureste de España. Acta Botánica Malacitana 21: 35-47.

ELDRIDGE D., FERRIS J. 1999. Recovery of Populations of the Soil Lichen Psora Crenata After Disturbance in Arid South Australia. The Rangeland Journal. 21:194 - 198

FERNÁNDEZ-GÁLVEZ J., BARAHONA E., MINGORANCE M. 2008. Measurement of Infiltration in Small Field Plots by a Portable Rainfall Simulator: Application to Trace-Element Mobility. Water Air Soil Pollution. 191:257-264.

GARCÍA-PALACIOS P., SOLIVERES S., MAESTRE F., ESCUDERO A., CASTILLO MONROY A., VALLADARES F. 2010. Dominant plant species modulate responses to hydroseeding, irrigation and fertilization during the restoration of semiarid motorway slopes. Ecological engineering. 36:1290-1298.

GIL C., RAMOS J.J. 2011. Los suelos yesíferos (Gipsisoles). En: J.F Mota., F. MartínezHernández \& J.S. Guirado (Eds.). Diversidad Vegetal de las Yeseras Ibéricas. El reto de los 
archipiélagos edáficos para la biología de la conservación: 261-262. ADIF-Mediterráneo Asesores Consultores. Almería.

GILBERT O.L. 1991. A successful transplant operation involving Lobaria amplissima. Lichenologist. 23:73-76

GREEN T.G.G., NASH III T.H., LANGE L. 2008. Physiological ecology of carbon dioxide exchange. En: T. H. Nash III T.H. Lichen Biology. Second edition: 152-181. Cambridge University Press, New York.

GRETTARSDOTTIR J., ARADÓTTIR A.L., VANDVIK V., HEEGAARD E., BIRKS H.J.B. 2004. Long term effects of reclamation treatments on plant succession in Iceland. Restoration Ecology. 12:268-278.

GUERRA J., ROS R.M., MARTÍNEZ-SÁNCHEZ J.J., DE LAS HERAS J., HERRANZ J.M., CANO M.J. 1993. Flora y vegetación briofítica de las zonas yesíferas de la provincia de Albacete. Al-Basit: Revista de estudios albacetenses 32: 39-62.

GUERRA J., ROS R.M. CANO M.J., CASARES M. 1995. Gypsiferous outcrops in SE Spain, refuges of rare, vulnerable and endangered bryophytes and lichens. Cryptogamie Bryologie Lichenologie 16: 125-135.

GUTIÉRREZ-CARRETERO L., CASARES-PORCEL M. 2011. Los líquenes en los afloramientos de yesos de la Península Ibérica. En: J. F Mota. , P. Sánchez- Gómez \& J.S Guirado. (eds.) 2011. Diversidad vegetal de la yeseras ibéricas. El reto de los archipiélagos edáficos para la biología de la conservación: 549-565. ADIFMediterráneo Asesores Consultores. Almería.

HARPER K. T., BELNAP J. 2001. The influences of biological soil crusts on mineral uptake by associated vascular plants. Journal of Arid Environments 47: 347-357.

HEIKEN T. 1999. Dispersal patterns of terricolous lichens by thallus fragments. Lichenologist 31(6): 603-612. 
HILTY J. 2004. Recovery of biological soils crusts following wildfire in Idaho. Rangeland Ecology \& Management 57: 89-96.

HOTHORN T., BRETZ F., WESTFALL P. 2008. Simultaneous inference in general parametric models. Biometrical Journal,50: 346-363.

IBARZ N. 2012. Primera experiencia de restauración de la costra liquénica en ambientes semiáridos. Memoria final Trabajo de Fin de Master. Universidad de Granada. Inédito.

JENSEN L.S., MUELLER T., TATE K.R., ROSS D.J., MAGID J. NIELSEN N.E. 1996. Soil surface $\mathrm{CO}_{2}$ flux as an index of soil respiration in situ: a comparison of two chamber methods. Soil Biology Biochemistry 28: 1297-1306.

KNAUF. 2008. Plan de explotación de las canteras de Escúzar y Ventas de Huelma. Documento interno. Inédito.

LANGE O. L. 2003. Photosinthetic productivity of the epilithic lichen Lecanora mralis: long-term field monitoring of $\mathrm{CO}_{2}$ eschange and its physiological interpretation. Flora 198: 277-292.

LANGE O. L., GREEN T. G. 2006. Nocturnal respiration of lichens in their natural habitat is not affected by preceding diurnal net photosynthesis. Oecologia 148: 396-404.

LANGE O.L. 2007. Water relations and carbon dioxide exchange of epiphytic lichens in the Namib fog desert. Flora 202: 479-487.

LI X. L., XIAO H.L., ZHANG J.G., WANG X.P. 2004. Long-term ecosystem effects of sandbinding vegetation in the Tengger Desert, northern China. Restoration Ecology. 12:376-390

LLIMONA X. 1974. Las comunidades de líquenes de los yesos de España. PhD. Tesis. Universitat de Barcelona, Barcelona. 
LORITE J., CAÑAdas E.M., BALlesteros M., PEÑAS J., VAlLe F. 2011. Yesos del Temple-La Malahá. En: J.F Mota., F. Martínez-Hernández, J.S. Guirado (Eds.). Diversidad Vegetal de las Yeseras Ibéricas. El reto de los archipiélagos edáficos para la biología de la conservación: 147-151. ADIF-Mediterráneo Asesores Consultores. Almería.

MARCHAL F.M., LENDÍNEZ M.L., SALAZAR C., TORRES J.A. 2008. Aportaciones al conocimiento de la vegetación gipsícola en el occidente de la provincia de Granada (sur de España). Lazaroa 29: 95-100.

MARTÍNEZ-SÁNCHEZ J.J. 1994. A special habitat for bryophytes and lichens in the arid zones of Spain. Lindbergia 19: 116-121.

MATESANZ S., VALLADARES F., TENA D., COSTA-TENORIO M., BOTE D. 2006. Early Dynamics of Plant Communities on Revegetated Motorway Slopes from Southern Spain: Is Hydroseeding Always Needed?. Restoration Ecology. 14:297-307.

MATESANZ S., VALLADARES F. 2007. Improving revegetation of gypsum slopes is not a simple matter of adding native species: Insights from a multispecies experiment. Ecological engineering 30: 67-77.

MERLIN G., DI-GIOIA L., GODDON C. 1999. Comparative study of the capacity of germination and of adhesion of various hydrocolloids used for revegetalization by hydroseeding. Land degradation and development. 10: 21-34.

MOORE P. D. 1998. Desert ecology: Life in the upper crust. Nature, 393: 419-420.

MOTA, J.F., SOLA A.J., JIMÉNEZ-SÁNCHEZ M.L., PÉREZ-GARCÍA F.J., MERLO M.E. 2004. Gypsicolous flora, conservation and restoration of quarries in the southeast of the Iberian Peninsula. Biodiversity and Conservation 13: 1797-1808. 
NASH, T.H. 1996. Lichen Biology. Cambridge University press. New York.

PALMQVIST K., DAHLMAN L., JONSSON A., NASH III T.H. 2008. The carbon economy of lichens. En T. H Nash III. 2008. Lichen Biology. Second edition: 182-215. Cambridge University Press, New York.

R DEVELOPMENT CORE TEAM. 2014. R: A language and environment for statistical computing. R Foundation for statistical computing, Vienna, Austria. Http://www.r-project.org

RIBA O., MACAU F. 1962. Situación, Características y Extensión de Los Terrenos Yesíferos en España. Actas del I Coloquio Internacional sobre las obras públicas en los terrenos yesiferos: 133.

RUBIO A., ESCUDERO A. 2000. Small-scale spatial soil-plant relationship in semi-arid gysum environments. Plant and Soil 220: 139-150.

SALAS, R. 2008. Estudio de impacto ambiental y propuesta de medidas correctoras de las canteras de Vental de Huelma y Escúzar. Informe Administrativo. Inédito.

SMITH D.C., MOLESWORTH S. 1973. Lichen physiology XIII. Effects of rewetting dry lichens. New Phytologist. 72:525-533.

SMITH P.L., 2014. Lichen translocation with reference to species conservation and habitat restoration. Symbiosis. 62:17-28.

THERNEAU T., 2013. A Package for Survival Analysis in S. R Package Version 2.37-4.

TORMO J., BOCHET E., GARCÍA-FAYOS P. 2007. Roadfill Revegetation in Semiarid Mediterranean Environments. Part II: Topsoiling, Species Selection, and Hydroseeding. Restoration Ecology. 15:97-102. 


\section{ANEXO 1}

En cuanto al material de rechazo utilizado en las simulaciones y presente en la experiencia de campo podemos decir que se trata de un material relativamente heterogéneo, con un contenido en grava entre el 30\% y el 39 \% con una composición mayoritaria de yeso. Tiene una textura de la fracción tierra fina $(<2 \mathrm{~mm})$ arcillo-limosa, con un contenido medio en limo superior al 50\% y en arcilla próximo al 40\%. El contenido en yeso oscila entre 93-25\%. Presenta valores muy bajos para el N $(0.029 \%)$ y materia orgánica $(0.07 \%)$, y bajos para K, Ca y Mg.

\begin{tabular}{|c|c|c|c|c|c|c|c|c|}
\hline & \multicolumn{6}{|c|}{ Análisis sustrato } & \multicolumn{2}{|c|}{ Análisis suelo } \\
\hline & Mínimo & Máximo & \begin{tabular}{|l|}
$\begin{array}{l}\text { Error } \\
\text { estándar }\end{array}$ \\
\end{tabular} & Media & & Clasificación(1) & Valores & Clasificación(1) \\
\hline Arena (\%) & 5.25 & 12.45 & 0.62 & 8.99 & \multirow{3}{*}{$\begin{array}{l}\text { Limo } \\
\text { total } \\
50.40\end{array}$} & \multirow{4}{*}{$\begin{array}{c}\text { Textura fina } \\
\text { Arcillo limosa }\end{array}$} & 27.6 & \multirow{4}{*}{ Textura franca } \\
\hline $\begin{array}{l}\text { Limo_gr } \\
(\%)\end{array}$ & 3.05 & 27.90 & 1.79 & 9.60 & & & \multirow{2}{*}{$\begin{array}{l}\text { Limo } \\
\text { total } \\
50.3\end{array}$} & \\
\hline Limo_f $(\%)$ & 31.27 & 59.68 & 2.19 & 41.44 & & & & \\
\hline Arcilla (\%) & 21.77 & 51.50 & 2.37 & 39.97 & & & 22.10 & \\
\hline Grava (\%) & 30.10 & 38.80 & 1.89 & 33.48 & & & 41.00 & \\
\hline Yeso (\%) & 24.54 & 92.86 & 8.14 & 47.96 & & & & \\
\hline $\mathrm{CaCO} 3(\%)$ & 22.55 & 31.81 & 1.02 & 27.25 & & Alto & 21.00 & Normal \\
\hline N (\%) & 0.022 & 0.040 & 0.00 & 0.029 & & Muy bajo & 0.05 & Bajo \\
\hline C_T (\%) & 2.77 & 3.80 & 0.13 & 3.26 & & $x$ & 3.07 & 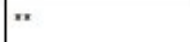 \\
\hline $\begin{array}{l}\text { C_inorg } \\
(\%)\end{array}$ & 2.71 & 3.82 & 0.12 & 3.27 & & $n$ & 2.52 & - \\
\hline $\operatorname{CO}(\%)$ & 0.01 & 0.09 & 0.01 & 0.04 & & 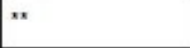 & 0.55 & $=$ \\
\hline MO (\%) & 0.01 & 0.15 & 0.02 & 0.07 & & Muy bajo & 0.64 & Bajo \\
\hline CE $\left(\mathrm{dS} \cdot \mathrm{m}^{-1}\right)$ & 2.25 & 2.29 & 0.00 & 2.27 & & Salinidad escasa & 3.10 & Salinidad escasa \\
\hline $\mathrm{pH}(\mathrm{H} 2 \mathrm{O})$ & 7.72 & 7.86 & 0.01 & 7.79 & & $\begin{array}{l}\text { Medianamente } \\
\text { básico }\end{array}$ & 7.60 & $\begin{array}{l}\text { Medianamente } \\
\text { básico }\end{array}$ \\
\hline $\begin{array}{l}\mathrm{Na} \\
\text { (cmol/kg) }\end{array}$ & 0.03 & 0.06 & 0.00 & 0.04 & & $x$ & 0.10 & 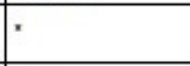 \\
\hline $\begin{array}{l}\mathrm{K} \\
(\mathrm{cmol} / \mathrm{kg})\end{array}$ & 0.12 & 0.32 & 0.02 & 0.20 & & Bajo & 0.20 & Bajo \\
\hline $\begin{array}{l}\mathrm{Mg} \\
(\mathrm{cmol} / \mathrm{kg})\end{array}$ & 0.16 & 0.31 & 0.01 & 0.22 & & Muy bajo & 0.66 & Bajo \\
\hline $\begin{array}{l}\mathrm{Ca} \\
\text { (cmol/kg) }\end{array}$ & 4.78 & 12.19 & 0.53 & 7.68 & & Bajo & 29.10 & Alto \\
\hline $\begin{array}{l}\mathrm{CIC} \\
(\mathrm{cmol} / \mathrm{kg})\end{array}$ & 5.33 & 12.51 & 0.51 & 8.15 & & * & 10.10 & Bajo \\
\hline $\operatorname{cc}(\%)$ & 29.13 & 32.58 & 0.41 & 31.09 & & 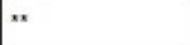 & 30.10 & $m$ \\
\hline PM (\%) & 20.14 & 22.89 & 0.34 & 20.98 & & 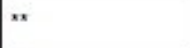 & 22.50 & $=$ \\
\hline$A U(\%)$ & 8.86 & 11.39 & 0.38 & 10.11 & & $\approx$ & 7.60 & ." \\
\hline
\end{tabular}

Tabla X. Resultados de la caracterización del sustrato y de suelo de la zona. (Abreviaturas de los parámetros analizados: Limo_gr (limo grueso), Limo_f (limo fino), N (nitrógeno), C_T (carbono total), C_inorg. (carbono inorgánico), CO (carbono orgánico), MO (materia orgánica), $\overline{\mathrm{C}}$ (conductividad eléctrica), $\mathrm{Na}$ (sodio), $\mathrm{K}$ (potasio), $\mathrm{Mg}$ (magnesio), Ca (calcio), CIC (capacidad reintercambio catiónico), CC (capacidad de campo), PM (punto de marchitamiento), AU (agua útil)). (Fuente: Trabajo Fin de Máster Raquel Aguilera Molina,UGR). 


\section{ANEXO 2}

En la tabla de abajo se muestran los datos de los picos de pluviometría por hora para los años 2010 y 2011. Se han eliminado aquellos datos que estaban por debajo de los $10 \mathrm{~L} / \mathrm{m}^{2}$. Los datos que resultaban más útiles para la experiencia de laboratorio eran los máximos de precipitación en un momento determinado.

\begin{tabular}{|c|c|c|c|c|c|}
\hline \multicolumn{6}{|c|}{ PICOS de INTENSIDAD de LLUVIA por HORAS } \\
\hline \multicolumn{3}{|c|}{2010} & \multicolumn{3}{|c|}{2011} \\
\hline Fecha & Hora & INTENSIDAD & Fecha & Hora & INTENSIDAD \\
\hline 2010_02_19 & $21: 33: 44$ & 15,20 & 2011_04_21 & 18:31:07 & 10,60 \\
\hline 2010_02_19 & $06: 33: 44$ & 25,70 & 2011_04_22 & 03:31:07 & 11,27 \\
\hline 2010_02_22 & $07: 33: 44$ & 11,20 & 2011_04_22 & 05:31:07 & 18,60 \\
\hline $2010 \_02 \_22$ & $09: 33: 44$ & 13,20 & 2011_04_23 & $12: 31: 07$ & 16,80 \\
\hline 2010_02_22 & $16: 33: 44$ & 21,30 & 2011_04_23 & 17:31:07 & 16,70 \\
\hline $2010 \_02 \_23$ & $06: 33: 44$ & 10,10 & 2011_04_24 & $20: 31: 07$ & 27,20 \\
\hline 2010_02_23 & $07: 33: 44$ & 13,70 & 2011_04_24 & $21: 31: 07$ & 16,20 \\
\hline 2010_02_27 & $18: 33: 44$ & 14,70 & 2011_05_02 & 01:31:07 & 51,40 \\
\hline 2010_03_02 & $22: 04: 15$ & 14,70 & 2011_05_02 & 19:31:07 & 13,50 \\
\hline 2010_03_02 & $23: 04: 15$ & 14,00 & 2011_05_02 & $21: 31: 07$ & 11,30 \\
\hline 2010_03_03 & $18: 04: 15$ & 10,10 & 2011_05_07 & $06: 31: 07$ & 14,20 \\
\hline 2010_03_07 & $23: 04: 15$ & 19,00 & 2011_05_07 & $07: 31: 07$ & 20,90 \\
\hline 2010_03_08 & 00:04:15 & 13,08 & 2011_05_07 & 08:31:07 & 36,60 \\
\hline 2010_03_08 & $02: 04: 15$ & 11,50 & 2011_05_07 & 09:31:07 & 57,20 \\
\hline 2010_03_08 & 03:04:15 & 17,50 & 2011_05_07 & $10: 31: 07$ & 28,20 \\
\hline 2010_03_08 & $22: 04: 15$ & 11,00 & 2011_05_07 & $15: 31: 07$ & 22,40 \\
\hline 2010_04_22 & $18: 31: 07$ & 18,80 & 2011_05_20 & 03:31:07 & 61,50 \\
\hline 2010_05_10 & $15: 31: 07$ & 18,90 & 2011_07_12 & $15: 37: 07$ & 11,60 \\
\hline 2010_05_11 & $01: 31: 07$ & 11,40 & 2011_10_24 & $11: 04: 29$ & 14,40 \\
\hline 2010_06_13 & $14: 17: 26$ & 38,20 & 2011_10_24 & $17: 04: 29$ & 28,40 \\
\hline 2010_06_13 & $15: 17: 26$ & 38,00 & 2011_10_24 & $18: 04: 29$ & 11,70 \\
\hline 2010_06_15 & $03: 17: 26$ & 89,70 & 2011_10_27 & $11: 04: 29$ & 26,70 \\
\hline 2010_06_29 & $19: 17: 26$ & 20,80 & 2011_10_27 & $20: 04: 29$ & 11,80 \\
\hline 2010_06_29 & $20: 17: 26$ & 17,20 & 2011_10_28 & $18: 04: 29$ & 28,10 \\
\hline 2010_07_03 & $05: 17: 26$ & 10,30 & 2011_11_03 & $06: 04: 29$ & 15,90 \\
\hline 2010_07_03 & $11: 17: 26$ & 25,50 & 2011_11_03 & $07: 04: 29$ & 19:30 \\
\hline \multirow[t]{8}{*}{ 2010_07_08 } & $23: 17: 26$ & 12,20 & 2011_11_03 & $08: 04: 29$ & 15,20 \\
\hline & & & 2011_11_03 & $14: 04: 29$ & 39,90 \\
\hline & & & 2011_11_03 & $16: 04: 29$ & 22,30 \\
\hline & & & 2011_11_04 & $03: 04: 29$ & 55,00 \\
\hline & & & 2011_11_04 & $09: 04: 29$ & 26,30 \\
\hline & & & 2011_11_04 & $10: 04: 29$ & 26,10 \\
\hline & & & 2011_11_04 & $16: 04: 29$ & 18,90 \\
\hline & & & 2011_11_08 & $00: 04: 29$ & 12,70 \\
\hline
\end{tabular}




\section{AGRADECIMIENTOS}

En primer lugar agradecer a mis tutores Juan y Manolo por su disponibilidad y atención siempre que la he requerido, sin su consejo y directrices hubiera sido muy complicado realizar este trabajo. Gracias a Paco por su ayuda y su buena disposición, su colaboración ha sido fundamental para que este trabajo salga adelante.

También muchas gracias a Eva por su ayuda cuando la he necesitado. Y en especial gracias a Miguel compañero de fatigas, paciente y atento a todas mis dudas e inquietudes, que no han sido pocas. 